\title{
On the Detection of a Class of Fast Frequency-Hopped Multiple Access Signals
}

\author{
Yu T. Su, Member, IEEE, Ye-Shun Shen, and Chu-Ya Hsiao
}

\begin{abstract}
The capacity of a fast frequency-hopped multiple access (FHMA) system is dictated by two major related design concerns: the hopping pattern and the receiver structure. This paper studies the impact of these two factors. We present a maximum likelihood (ML) diversity combiner for detecting asynchronous FHMA multilevel FSK (MFSK) signals in Rician fading channels and analyze the performance of a close approximation of the ML receiver. We compare systems using random hopping patterns and those using optimal hopping patterns of Einarsson [8]. Performance comparisons between chip-synchronous and chip-asynchronous systems are made as well. We propose and examine the effectiveness of a two-stage multiuser detector, in which the first stage makes an initial decision while the second stage tries to reduce multiple access interference (MAI) and resolve the ambiguity left by the first stage detector. The MAI caused by undesired users is constituted by a cochannel interference (CCI) contribution and an interchannel interference (ICI) contribution. This detector is of modest complexity and is capable of removing most of the CCI and part of the ICI. Two methods for mitigating MAI are also examined.
\end{abstract}

Index Terms-Frequency hop communications, multiaccess communications, signal detection.

\section{INTRODUCTION}

$\mathbf{T}$ HE DETECTION of a code division multiple access (CDMA) signal is often hampered by the interference generated by system users whose carrier frequencies are the same [i.e., cochannel interference (CCI)] or close to [interchannel interference (ICI)] that used by the desired user. Therefore, in order to enhance the signal's detectability and increase the system's capacity, one needs to devise a detection scheme for reducing both the CCI and ICI. This paper suggests several detector structures for the fast frequency-hopped multiple access systems studied in [1]-[10]. We also examine their efficiency in dealing with the above-mentioned interference and evaluate the related system capacity as well as spectral efficiency.

Earlier analysis on noncoherent frequency-hopped multiple access (FHMA) systems [1]-[6] assume that: 1) a single-stage detector is employed by the receiver and 2) random memoryless FH patterns (users' addresses) are used. Moreover, except

Manuscript received December 30, 1999; revised June 18, 2000. This work was supported in part by the National Council of Taiwan under Grant NSC 84-2612E009-003. This work was presented in part at the VTC'96, Atlanta, GA, USA.

Y. T. Su is with the Department of Communication Engineering, National Chiao Tung University, Hsinchu 30056, Taiwan R.O.C.

Y.-S. Shen is with the International Business Group, Chunghwa Telecom, Kaohsiung 80053, Taiwan R.O.C.

C.-Y. Hsiao is with Novatek Microelectronics, Hsinchu 30077, Taiwan R.O.C.

Publisher Item Identifier S 0733-8716(01)09081-3. for [3], they also assume that a chip(hop)-synchronous mode is maintained, i.e., all users switch their carrier frequencies simultaneously. Since multilevel FSK (MFSK) is used in these systems, 1) implies that the detector will simply choose the channel exhibiting the largest combined value as its output and 2) tells us that the performance obtained in this analysis is actually the performance averaged over all possible address assignment schemes. The third (chip-synchronous) assumption is a more practical assumption for the forward link than for the reverse link. This assumption also exempts the receiver from dealing with ICI, if an appropriate channel separation is used by the system. Removal of this assumption, as we shall see, will lead to serious ICI that may substantially impair a receiver's detection capability.

Solomon [7] and Einarsson [8] had proposed FH pattern assignment schemes, which render minimum MA interference. Taking advantage of the inherent algebraic structure of these constructions, Timor [9] presented a two-stage chip-synchronous demodulator that is capable of eliminating most of the CCI if the thermal noise effect can be neglected. He later [10] suggested a multistage decoding algorithm to further improve the performance of the two-stage algorithm. Mabuchi et al. [11] proposed a class of multistage demodulation algorithms for FHMA/MFSK systems, which is similar to the multistage scheme developed in [10]. These algorithms, like that described in [10], are much more complicated than the two-stage decoding algorithm [9], since they require first-stage detection of all users. According to these studies [9]-[11], multiuser detection of fast FHMA/MFSK signals can be decomposed into two major successive stages. The first stage combines noncoherently detected outputs matched to various chips (hops) in an appropriate manner and makes a decision if possible, while the second stage attempts to resolve the ambiguity (if exists) left by the first stage of the detector. The first stage can reduce $\mathrm{CCI}$ and ICI but is incapable of eliminating them. The CCI and ICI cancellation is accomplished mostly by the second stage. Furthermore, the second stage detection can be divided into several substages, depending on whether outputs from other users' detectors are available.

Our effort reported in this paper can be summarized as follows. After a brief description of the system under investigation in Section II, we derive a nearly maximum likelihood (ML) single stage detector for asynchronous FHMA/MFSK systems in Section III and analyze the performance of a receiver that is a close approximation of the ML one. In Section IV, we examine the performance of both the synchronous and asynchronous systems using the optimal hopping pattern suggested by Einarsson. Means for containing the MA interference are suggested and 


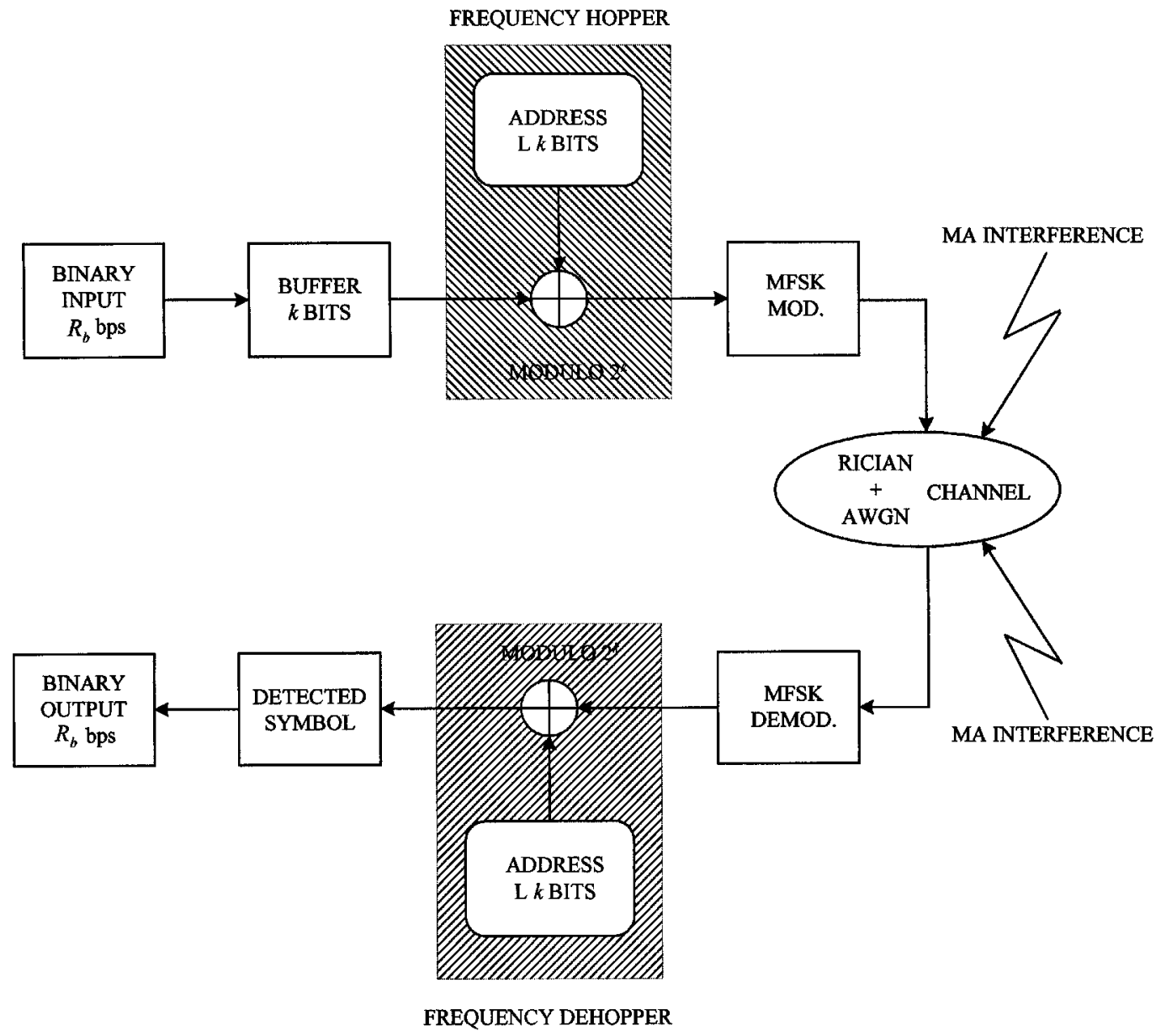

Fig. 1. A block diagram for the FHMA/MFSK system under investigation.

the associated statistical properties are derived in this section as well. Section V proposes a two-stage multiuser detection scheme while Section VI summarizes the major results and observations obtained in this contribution. The Appendixes present derivations of some key equations and a modified interference test.

\section{SYSTEM DESCRIPTION AND DEFINITIONS}

A block diagram of the FHMA/MFSK system proposed by Goodman et al. [1] is depicted in Fig. 1. The data source produces a data bit stream of rate $R_{b}$ bits/s. The data stream is mapped into an MFSK signal sequence of rate $R_{s}$ symbols/s, where $R_{s}=1 / T_{s}=1 /\left(k T_{b}\right)$ and $k=\log _{2} M$. The carrier frequency of an MFSK symbol is then hopped $L$ times within $T_{s}$ s, i.e., the subsymbol (chip) duration is $T_{c}=T_{s} / L \mathrm{~s}$ and the chip rate is $R_{c}=1 / T_{c}$. Suppose $W(\mathrm{~Hz})$ is the hopping bandwidth and the channel spacing is $\Delta f=R_{c} \mathrm{~Hz}$, then the channel (tone) number $N_{t}$ available for hopping is given by

$$
N_{t}=\left\lfloor W / R_{c}\right\rfloor=\left\lfloor W k / L R_{b}\right\rfloor
$$

where $\lfloor x\rfloor$ is the integer part of $x$ and $N_{t}$ is usually larger than $M$. In this paper, however, we will only consider the case of $M=N_{t}$, for such a system yields a spectral efficiency higher than those having other frequency structures of $M<N_{t}$ [6].
Thus the candidate values of $(M, L)$ for a given $W / R_{b}$ are related by

$$
\frac{M}{\log _{2} M}=\frac{W}{R_{b}} L^{-1}
$$

and the spectral efficiency $\beta$, measured in bits $/ \mathrm{s} / \mathrm{Hz}$, is given by [4]

$$
\beta=\frac{K_{\max } R_{b}}{W}=\frac{K_{\max } \log _{2} M}{M L}
$$

where $K_{\max }$ is the system capacity, i.e., the maximum number of active users the system can support while guaranteeing a given performance. When the channel spacing is wider than the minimum required value, i.e., when $\Delta f=\eta R_{c}$, $\eta>1$, then the spectral efficiency should be calculated by $\beta=K_{\max } \log _{2} M /(\eta M L)$. It is clear that a system's spectral efficiency can be improved by using a wider channel spacing, only if it brings a large enough capacity increase.

Frequency hopping and dehopping, as illustrated in Fig. 2, is performed in a deterministic manner. Each user is given an address (hopping pattern) which is an $L$-tuple, $\mathbf{a}=$ $\left(a_{0}, a_{1}, \ldots, a_{L-1}\right)$, where $a_{i} \in G, G=\{0,1, \ldots, M-$ $1\} \stackrel{\text { def }}{=} G F(M)$, represents a candidate MFSK tone of duration $T_{c}$ s. The actual transmitted frequencies within a symbol interval is $\mathrm{f}_{\mathbf{m}}=\left(b_{m 0}, b_{m 1}, \ldots, b_{m(L-1)}\right)=\mathbf{m} \oplus \mathbf{a}$, where $\mathbf{m}=(m, m, \ldots, m)$ is a constant $L$-tuple representing a $k$ 

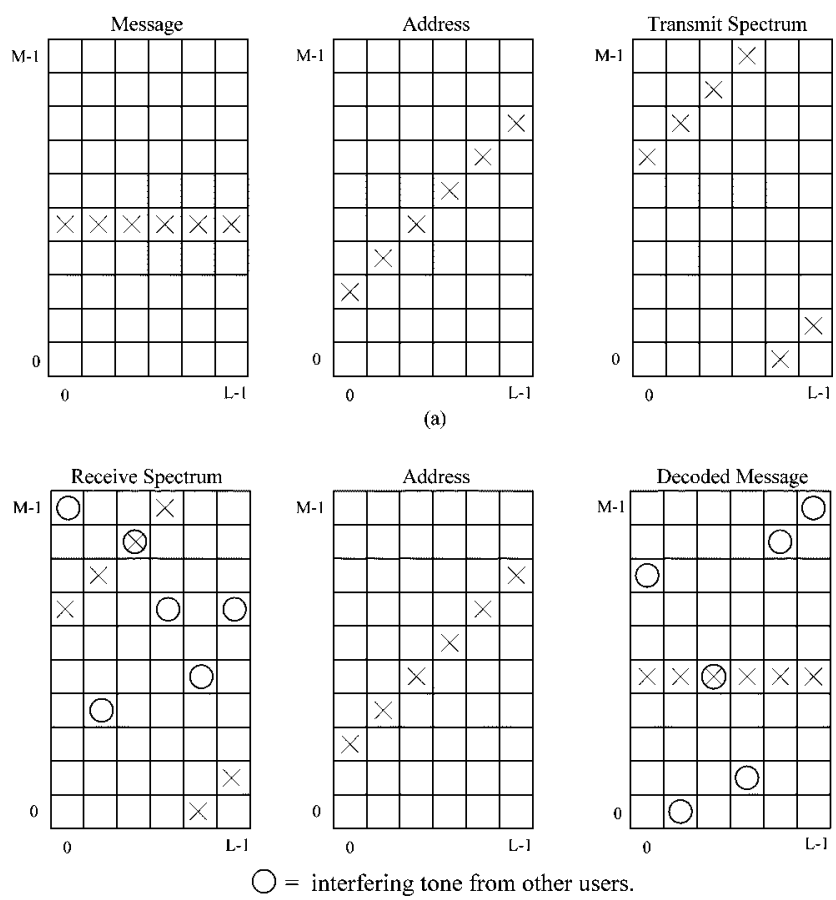

(b)

Fig. 2. Time-frequency matrices for (a) the transmitter and (b) the receiver.

bits message, $\oplus$ denotes modulo- $M$ addition, and $b_{m l} \in G$. An optimal assignment scheme suggested in [8] associates the $j$ th user an element $\lambda_{j} \in G F(M)$ and the address

$$
\mathbf{a}_{j}=\left(\lambda_{j}, \lambda_{j} \beta, \lambda_{j} \beta^{2}, \ldots, \lambda_{j} \beta^{L-1}\right)
$$

where $\beta$ is a fixed primitive element in $G F(M)$.

Since the transmitted signal, $\hat{s}_{m}(t)$, is required to hop within the frequency band $\left(f_{0}, f_{0}+W\right)=\left(f_{0}, f_{0}+M / T_{c}\right)$, where $f_{0}$ is the lowest frequency in the time-frequency (T-F) matrix as shown in Fig. 2, it can be expressed as

$$
\hat{s}_{m}(t)=\sum_{l=0}^{L-1} x_{b_{m l} l}(t) e^{i \psi_{m l}}
$$

where the random phases $\left\{\psi_{m l}\right\}$ result from the noncoherent frequency hopper. Furthermore, $x_{n l}(t), n=0,1, \ldots, M-1$, $l=0,1, \ldots, L-1$ is a set of basis waveforms defined by

$$
x_{n l}(t)=\sqrt{S} P_{T_{c}}\left(t-l T_{c}\right) e^{i\left[2 \pi\left(f_{0}+n \Delta f\right) t\right]}
$$

$S=E_{c} / T_{c}$ is the signal power, $E_{c}$ is the average signal energy per chip, and

$$
P_{T_{c}}(t)= \begin{cases}1, & 0 \leq t<T_{c} \\ 0, & \text { otherwise }\end{cases}
$$

The received waveform is first dehopped and then detected by a bank of energy detectors as shown in Fig. 3. Typical received and dehopped spectrums are shown in the bottom half of Fig. 2, where circles in the T-F matrix are interfering tones from other users. The received waveform, corresponding to the desired user, consists of three components

$$
\hat{r}(t)=\hat{c}_{m}(t)+\sum_{j=1}^{J} \hat{I}_{j}(t)+n(t)
$$

where

$$
\hat{c}_{m}(t)
$$

$\hat{I}_{j}(t) \quad$ interference caused by the $j$ th interferer;

$J$ number of interferers (hence there are $K=J+1$ users in the system);

$n(t) \quad$ additive white Gaussian noise (AWGN) whose onesided power spectral density is $N_{0} \mathrm{~W} / \mathrm{Hz}$.

We model the channel effect as such that all candidate basis waveforms $\left\{x_{n l}(t)\right\}$ suffer from frequency nonselective fading obeying an independent and identically Rician distribution. More specifically, we assume that different MFSK tones at the same chip experience independent and identically distributed (i.i.d.) frequency nonselective Rician fading and statistics of a user signal at different chips are i.i.d. as well. The former (tone-independent) assumption is appropriate, since different user signals-even if the same carrier frequency are employed in the same interval-are propagated over different physical paths. The second (chip-independent) assumption is approximately valid, because it is either impossible [7], [8] or unlikely (when a set of random hopping patterns with large $M$ is used) for neighboring chips of a user address to use the same frequency or even the adjacent frequencies. Ignoring the propagation delay, the desired signal in the first symbol period can therefore be written as

$$
\hat{c}_{m}(t)=\sum_{l=0}^{L-1} \alpha_{m l} \sqrt{S} P_{T_{c}}\left(t-l T_{c}\right) e^{i\left[2 \pi\left(f_{0}+b_{m l} \Delta f\right) t+\theta_{m l}\right]}
$$

where $\left\{\theta_{m l}\right\}$ are random variables (r.v.s) uniformly distributed within the interval of $[0,2 \pi)$ and $\left\{\alpha_{m l}\right\}$ are i.i.d. Rician r.v.s. Note that $\left\{\theta_{m l}\right\}$ represent the noncoherent receiver's uncertainty about the phase of the received waveform. This uncertainty is the sum of the random phases associated with the diffused component (induced by the channel) and the specular component [i.e., $\left\{\psi_{m l}\right\}$ of (5)] of the received waveform. Given the phase of the transmitter $\psi_{m l}$, the phase of the diffused component follows an unimodal distribution and is correlated with $\alpha_{m l}$. However, after taking the average over $\psi_{m l}$, which is uniformly distributed within $[0,2 \pi)$, we can show that $\theta_{m l}$ is also uniformly distributed within $[0,2 \pi)$ and is independent of $\alpha_{m l}$. Let $\Gamma$ be the ratio of the power $a^{2}$ in the specular component to the power, $2 \sigma_{f}^{2}$, in the diffused component. Using the normalization, $E\left(\alpha_{m l}^{2}\right)=a^{2}+2 \sigma_{f}^{2}=1$, we obtain $a^{2}=\Gamma /(1+\Gamma)$ and $2 \sigma_{f}^{2}=1 /(1+\Gamma)$, respectively. When $\Gamma=0$ (no direct path) we have flat Rayleigh fading and $\Gamma=\infty$ (no diffused part) represents the AWGN-only case.

The $j$ th interfering signal, being transmitted through a similar but independent Rician fading channel, has the form

$$
\begin{aligned}
\hat{I}_{j}(t)= & \sum_{k=0}^{M-1} \sum_{l=0}^{L-1} \alpha_{j l} c_{j k l} \sqrt{S} P_{T_{c}}\left[t-\left(l-\epsilon_{j}\right) T_{c}\right] \\
& \cdot e^{i\left[2 \pi\left(f_{0}+k \Delta f\right) t+\theta_{j l}\right]}
\end{aligned}
$$




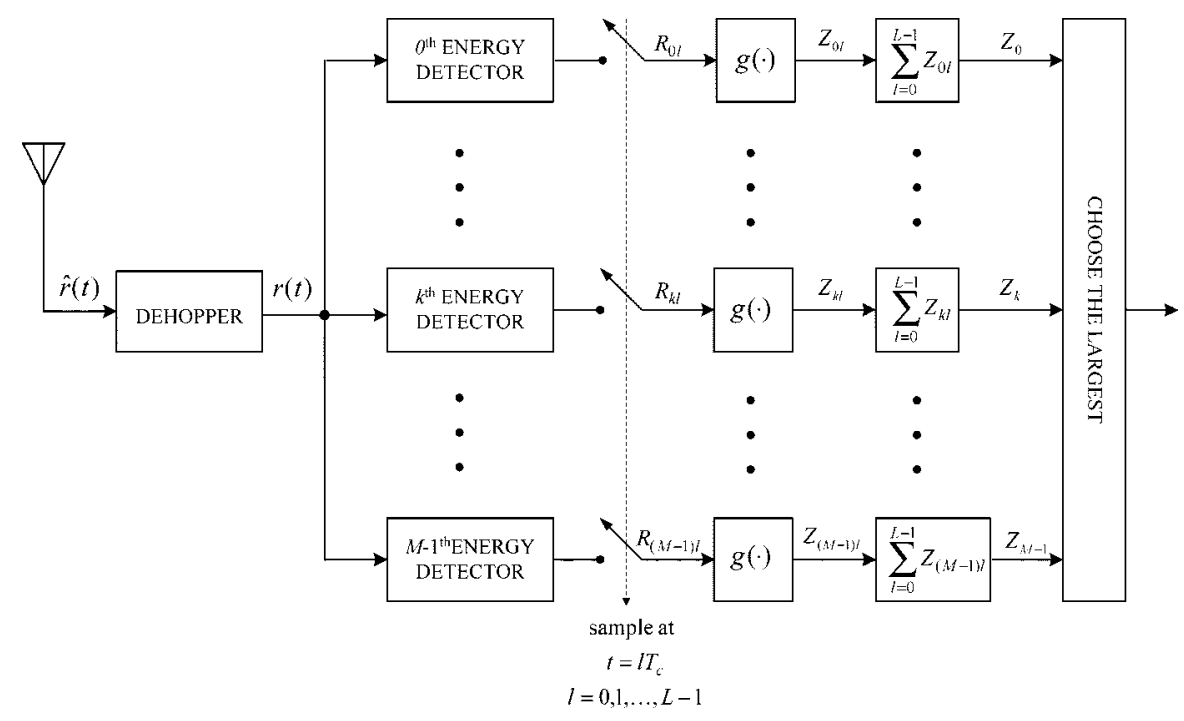

Fig. 3. A nonlinear diversity-combining receiver for noncoherent FHMA/MFSK signals.

where $\left\{\alpha_{j l}\right\}$ and $\left\{\theta_{j l}\right\}$ are independent random variables having the same distributions as $\left\{\alpha_{m l}\right\}$ and $\left\{\theta_{m l}\right\}$ of the desired signal. For each $(j, l)$ pair, however, only one $c_{j k l l}$ is nonzero, i.e., $c_{j k l}=\delta\left(k-b_{j l}\right)$, where $\delta(x)=0$ for all $x \neq 0$ and $\delta(0)=1$, $b_{j l}$ is the frequency tone used by the $j$ th interferer during the $l$ th chip interval. $\epsilon_{j}$ is the corresponding normalized timing misalignment with respect to the desired signal and is assumed to be uniformly distributed within $(0,1]$. For chip-synchronous systems, we have $\epsilon_{j}=0$ for all $j$.

With the received waveform given by (8)-(10), the dehopped signal becomes

$$
r(t)=c_{m}(t)+\sum_{j=1}^{J} I_{j}(t)+n(t)
$$

where

$$
\begin{gathered}
c_{m}(t)=\sum_{l=0}^{L-1} \alpha_{m l} \sqrt{S} P_{T_{c}}\left(t-l T_{c}\right) e^{i\left[2 \pi\left(f_{0}+m \Delta f\right) t+\phi_{m l}\right]} \\
I_{j}(t)=\sum_{k=0}^{M-1} \sum_{l=0}^{L-1}\left\{\alpha_{j l} c_{j k l} \sqrt{S} P_{T_{c}}\left[t-\left(l-\epsilon_{j}\right) T_{c}\right]\right. \\
\cdot e^{i\left[2 \pi\left(f_{0}+g_{k l} \Delta f\right) t+\phi_{j l}\right]} \\
+\alpha_{j(l+1)} c_{j k(l+1)} \sqrt{S} P_{T_{c}} \\
\cdot\left[t-\left(l+1-\epsilon_{j}\right) T_{c}\right] \\
\left.\cdot e^{i\left[2 \pi\left(f_{0}+g_{k l} \Delta f\right) t+\phi_{j(l+1)}^{\prime}\right]}\right\}
\end{gathered}
$$

and $g_{k l}=\left(k-a_{l}\right) \bmod M$. Since the phase of the local frequency synthesizer is not continuous (noncoherent) when it hops from one band to another, i.e., the "local phases" at different hops are independent, the dehopped random phases, $\phi_{m l}, \phi_{j l}, \phi_{j l}^{\prime}$, are i.i.d. r.v.s that are uniformly distributed within $[0,2 \pi)$.

The above equations, (11)-(13), imply that perfect power control has been assumed so that all the received signals have the same average strength. In the absence of channel perturbation, frequency dehopping followed by hard-decision detection is equivalent to a modulo- $M$ subtraction. Thus the message chips will fill a complete row in the receiver's T-F matrix whose index is equal to the desired message $\mathbf{m}$; see Fig. 2(b). The presence of the channel impairment may not only prevent the receiver from forming a complete row but also produce false rows [1], [9].

When hard-limited detection is applied to each diversity branch, the majority vote method that selects the row with the largest number of detected chips as the desired message is a simple and reasonable detection rule. In fact, this detection scheme is equivalent to a decision rule that chooses the codeword having minimum Hamming distance from the hard-limited detected word. If there are two or more rows having equal minimum distance, one of them will be selected randomly in order to remove the ambiguity. But the probability that hard-limited combining results in more than one largest row is an increasing function of the number of users $K$. These ambiguous events eventually become the dominant factor in limiting the system capacity. In general, there are two methods to resolve this kind of ambiguity. One is to use soft-decision schemes [6] while the other uses the information of the other users' addresses to eliminate the faulty entries in the receiver's T-F matrix. The first approach is studied in the following two sections while one alternative to the second approach will be presented in Section V.

\section{RANDOM MEMORYLESS FH PATTERN SYSTEM}

This section considers asynchronous random FH pattern systems. The interfering signals in (13) are assumed to have used mutually independent random and memoryless FH patterns (addresses). In other words, each component of a user's address is randomly and independently selected from the set $G$. Thus each element of the desired T-F matrix is hit by an interferer with probability $\mu \stackrel{\text { def }}{=} 1 / M$ if the chip-synchronous mode is assumed. Note that the multiple i.i.d. Rician channel assumption that leads to (8)-(10) and therefore (11)-(13) implies $\left\{\alpha_{j l}\right\}$, $\left\{\phi_{j l}\right\}$, and $\left\{\phi_{j l}^{\prime}\right\}$ are i.i.d. r.v.s. Furthermore, we emphasize that, as mentioned before, this assumption is only approximately 
true. Hence the claims of maximum likelihood and optimal nonlinearity in the following section are to be understood as such.

\section{A. Maximum Likelihood Receiver}

Let us first assume that the a priori probability of transmitting any bin is the same, i.e., $1 / M$. Denote the output of the $n$th energy detector at the $l$ th diversity branch (hop) during a given symbol interval (see Fig. 3) as $R_{n l}$ and the associated conditional probability density function (pdf) given that the $m$ th message bin was sent as $f_{R_{n l}}(R \mid m)$. By invoking the approximation that $\left\{R_{n l}\right\}$ are i.i.d. [3]-[6] and following a procedure similar to that presented in [3] and [6], we can show that the (nearly) optimal noncoherent Bayes' decision rule is given by

$$
\begin{gathered}
\text { Accept the hypothesis that } \\
\text { the } m \text { th tone was transmitted }\left(H_{m}\right) \text { if } \\
\sum_{l=0}^{L-1} \ln \left\{\frac{f_{R_{m l}}(R \mid m)}{f_{R_{m l}}(R \mid k)}\right\} \geq \sum_{l=0}^{L-1} \ln \left\{\frac{f_{R_{k l}}(R \mid k)}{f_{R_{k l}}(R \mid m)}\right\}, \quad \forall k \neq m .
\end{gathered}
$$

It can be shown that $R_{n l}$ is the squared magnitude of the complex random variable $U_{n l}$, which is given by

$$
\begin{aligned}
U_{n l} & =\frac{1}{E_{c}} \int_{l T_{c}}^{(l+1) T_{c}} r(t) x_{n l}^{*}(t) d t \\
& =s_{n l}+\sum_{j=1}^{J} I_{j n l}+z_{n l}
\end{aligned}
$$

where

$$
\begin{array}{ll}
r(t) & \text { is given by (11); } \\
x_{n l}^{*}(t) & \text { complex conjugate of } x_{n l}(t) \text { defined by } \\
& (6) ; \\
s_{n l}, I_{j n l}, \text { and } z_{n l} & \begin{array}{l}
\text { components contributed by the desired } \\
\text { signal, the } j \text { th interferer and AWGN, } \\
\text { respectively. }
\end{array}
\end{array}
$$

In particular, $z_{n l}$ is a zero-mean complex Gaussian r.v. whose real and imaginary parts have the same variance $\sigma_{0}^{2}=\left(2 E_{c} / N_{0}\right)^{-1}$. Substituting (6) and (11)-(13) into the upper part of (15), we immediately have

$$
s_{n l}=\alpha_{m l} e^{i \phi_{m l} l} \operatorname{sinc}[(m-n) \eta] e^{i[2 \pi(m-n)(l+1 / 2) \eta]}
$$

where $\operatorname{sinc}(x) \stackrel{\text { def }}{=} \sin (\pi x) / \pi x$, and

$$
\begin{gathered}
I_{j n l}=\sum_{k=0}^{M-1}\left\{\alpha_{j l} c_{j k l} e^{i \phi_{j l}}\left(1-\epsilon_{j}\right) \operatorname{sinc}\left[g_{k \ln }\left(1-\epsilon_{j}\right) \eta\right]\right. \\
\left.\cdot e^{i\left[2 \pi g_{k l n}\left(l+\left(1-\epsilon_{j} / 2\right)\right) \eta\right]}\right\} \\
+\sum_{k=0}^{M-1}\left\{\alpha_{j(l+1)} c_{j k(l+1)} \cdot e^{i \phi_{j(l+1)}^{\prime}} \epsilon_{j} \operatorname{sinc}\left[g_{k \ln } \epsilon_{j} \eta\right]\right. \\
\left.\cdot e^{i\left[2 \pi g_{k} \ln \left(l+1-\left(\epsilon_{j} / 2\right)\right) \eta\right]}\right\}
\end{gathered}
$$

where $g_{k l n}=g_{k l}-n=\left[\left(k-a_{l}\right) \bmod M\right]-n$.

The magnitude of the product $g_{k l n} \Delta f^{1}$ represents the frequency separation between the dehopped $j$ th interferer at the $l$ th

${ }^{1}$ Note that our interference model (10) allows only one nonzero $c_{j k l l}$ for each $(j, l)$ pair, hence only the corresponding $g_{k: \ln }$ does not vanish. hop and the $n$th energy detector. The term $\operatorname{sinc}\left[g_{k l n}\left(1-\epsilon_{j}\right) \eta\right]$ represents the contribution to the output of the $n$th energy detector associated with the $k$ th MFSK tone, which is generated by the $j$ th interferer in the $l$ th chip interval. According to (17) we can see that the orthogonality among the MFSK tones can no longer be maintained, since $\operatorname{sinc}\left[g_{k l n}\left(1-\epsilon_{j}\right) \eta\right]$ or $\operatorname{sinc}\left(g_{k \ln } \epsilon_{j} \eta\right)$ is nonzero unless $\epsilon_{j}=0$ or 1 for all $j$, and, simultaneously $\eta=\Delta f / R_{c}$ is an integer. When the latter conditions hold, (15) becomes

$$
U_{n l}=\alpha_{m l} \delta_{n m} e^{i \phi_{m l}}+\sum_{j=1}^{J} \alpha_{j l} c_{j n l} e^{i \phi_{j l}}+z_{n l}
$$

where $\delta_{n m}$ is the Kronecker delta function. In short, unless perfect chip synchronization can be achieved and the tone spacing equals an integer multiple of the chip rate, an MFSK tone transmitted by one user will impose ICI on all other FSK channels, hence on other active users' detectors.

A closer look at (15)-(17) reveals that the magnitude of $R_{n l}$ is a function of the basis waveform (6) because $\operatorname{sinc}^{2}(x)$ represents the energy spectrum value of a rectangular-waveformmodulated carrier at a certain frequency. It is possible to select a proper set of basis waveforms that minimize ICI. But this can only be accomplished at the expense of greater CCI, larger tone spacing, or longer symbol duration. It is very difficult to quantify the impact of the baseband design on the system performance. We claim no optimal property of (6) though we have examined several other nonrectangular baseband waveforms (like Barker-coded, square-root raised-cosine, Hamming-weighted, and Gaussian waveforms) and found no improvement in terms of spectral efficiency.

Although the channel separation $g_{k \ln }$ can be as large as $M-$ 1 , the corresponding ICI is a decreasing function of this parameter. Hence, we will only consider $2 B$ adjacent channels around the desired tone ( $B$ channels above and another $B$ channels below the desired one in the receiver T-F matrix) in the following analysis when examining the effect of ICI. An appropriate value for $B$ can be found via numerical evaluation of the associated performance (see Fig. 5).

For the multiple i.i.d. Rician fading channel, the relationship of $R_{n l}=X_{n l}^{2}=\left|U_{n l}\right|^{2}$ implies that the conditional pdf and cumulative distribution function (cdf) of $R_{n l}$ are given by (see Appendix A and [12] and [13])

$$
\begin{aligned}
& f_{R_{n l}}(R \mid m)=\frac{1}{2} \int_{0}^{\infty} \lambda J_{0}(\sqrt{R} \lambda) \Phi_{X_{n l}}(\lambda \mid m) d \lambda \\
& F_{R_{n l l}}(R \mid m)=\sqrt{R} \int_{0}^{\infty} J_{1}(\sqrt{R} \lambda) \Phi_{X_{n l}}(\lambda \mid m) d \lambda .
\end{aligned}
$$

In Appendix A, we show that the characteristic function of $X_{n l}$ is given by

$$
\begin{aligned}
\Phi_{X_{n l}}(\lambda \mid m) & e^{-\lambda^{2} \sigma_{0}^{2} / 2} e^{-\lambda^{2} \sigma_{f}^{2} \delta_{n m} / 2} J_{0}\left(a \lambda \delta_{n m}\right) \\
& \cdot \prod_{\rho=-B}^{B}\left[(1-\mu)^{2}+2 \mu(1-\mu) H(\lambda, \rho)+\mu^{2} G(\lambda, \rho)\right]^{J}
\end{aligned}
$$


where

$$
\begin{aligned}
H(\lambda, \rho)= & \int_{0}^{1} e^{-\epsilon^{2} \operatorname{sinc}^{2}\left(\rho \epsilon \lambda^{2} \sigma_{f}^{2} / 2\right.} J_{0}(a \epsilon \operatorname{sinc}(\rho \epsilon) \lambda) d \epsilon \quad(22 \mathrm{a}) \\
G(\lambda, \rho)= & \int_{0}^{1} e^{-\left\{(1-\epsilon)^{2} \operatorname{sinc}^{2}(\rho(1-\epsilon))+\epsilon^{2} \operatorname{sinc}^{2}(\rho \epsilon)\right\} \lambda^{2} \sigma_{f}^{2} / 2} \\
& \cdot J_{0}(a(1-\epsilon) \operatorname{sinc}[\rho(1-\epsilon)] \lambda) J_{0}(a \epsilon \operatorname{sinc}(\rho \epsilon) \lambda) d \epsilon .
\end{aligned}
$$

If the tone spacing is greater than the minimum required, i.e., if $\Delta f=\eta R_{c}, \eta>1$, then a procedure similar to that presented in Appendix A yields

$$
\begin{aligned}
\Phi_{X_{n l}}(\lambda \mid m) & \\
= & e^{-\lambda^{2} \sigma_{0}^{2} / 2} M(\lambda, m) \prod_{\rho=-B}^{B} \\
& \cdot\left[(1-\mu)^{2}+2 \mu(1-\mu) H(\lambda, \rho)+\mu^{2} G(\lambda, \rho)\right]^{J}
\end{aligned}
$$

where

$$
\begin{aligned}
M(\lambda, m)= & \begin{cases}\prod_{\substack{\rho=-B \\
\rho \neq 0}}^{B}\left\{1-\mu+\mu e^{-\operatorname{sinc}^{2}(\rho \eta) \lambda^{2} \sigma_{f}^{2} / 2}\right. & m \neq n \\
e^{-\lambda^{2} \sigma_{f}^{2} / 2} J_{0}(a \lambda), & m=n\end{cases} \\
H(\lambda, \rho)= & \int_{0}^{1} e^{-\epsilon^{2} \operatorname{sinc}^{2}(\rho \epsilon \eta) \lambda^{2} \sigma_{f}^{2} / 2} J_{0}[a \epsilon \operatorname{sinc}(\rho \epsilon \eta) \lambda] d \epsilon \\
G(\lambda, \rho)= & \int_{0}^{1} e^{-\left\{[1-\epsilon]^{2} \operatorname{sinc}^{2}[\rho(1-\epsilon) \eta]+\epsilon^{2} \operatorname{sinc}^{2}(\rho \epsilon \eta)\right\} \lambda^{2} \sigma_{f}^{2} / 2} \\
& \cdot J_{0}(a[1-\epsilon] \operatorname{sinc}[\rho(1-\epsilon) \eta] \lambda) \\
& \cdot J_{0}(a \epsilon \operatorname{sinc}[\rho \epsilon \eta] \lambda) d \epsilon .
\end{aligned}
$$

\section{B. A Suboptimal Realization}

The noncoherent Bayes receiver of (14) is depicted in Fig. 3 where the nonlinearity used in the receiver $g(x)$ is given by

$$
g(x)=\frac{R_{1 / 2}\left[g_{\mathrm{opt}}(x)-g_{\mathrm{opt}}(0)\right]}{g_{\mathrm{opt}}\left(R_{1 / 2}\right)-g_{\mathrm{opt}}(0)}, \quad 0<x<\infty
$$

with

$$
g_{\mathrm{opt}}(x) \stackrel{\text { def }}{=} \ln f_{R_{m l}}(x \mid m)-\ln f_{R_{m l}}(x \mid k) .
$$

Note that $f_{R_{m l}}(x \mid k)$ is the same for all $k \neq m$ and the value of $g_{\text {opt }}(x)$ is in fact only dependent on $x$ and not on $k$ or $m$ additionally. Furthermore, $R_{1 / 2}$ is derived according to $g_{\text {opt }}\left(R_{1 / 2}\right)=0.5 g_{\text {opt }}\left(R_{\text {sat }}\right)$, where the saturation point $R_{\text {sat }}$ is defined by $R_{\text {sat }}=\min \left\{x: g_{\text {opt }}^{\prime}(x) \stackrel{\text { def }}{=}(d / d x) g_{\text {opt }}(x)=0\right\}$. The normalized form of $g_{\text {opt }}(x),(25)$, is similar to $f(R)$ of [2] with the approximation, $\left[g_{\mathrm{opt}}\left(R_{1 / 2}\right)-g_{\mathrm{opt}}(0)\right] / R_{1 / 2} \approx$ $g_{\text {opt }}^{\prime}(0)$, and results in $g^{\prime}(0) \approx 1$. Such a normalization is useful for comparing the optimal nonlinearity under various

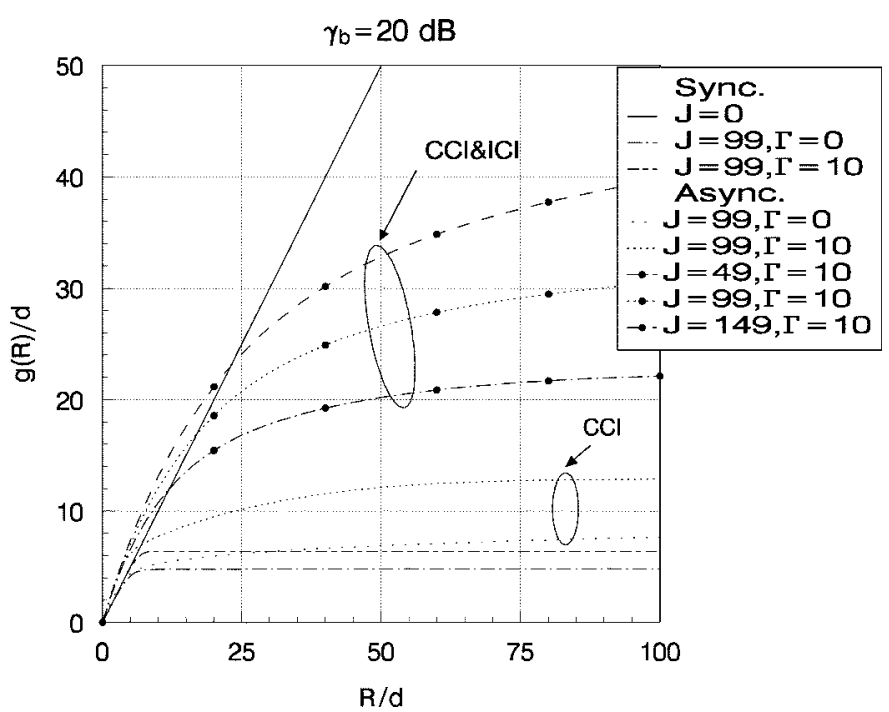

Fig. 4. The normalized optimal nonlinearity for both chip-asynchronous and synchronous modes in dependence of the number of interferers $J$, and the ratio $\Gamma$ between the specular and the diffused channel power, for a fixed SNR $\gamma_{b}=$ $20 \mathrm{~dB}$. Three curves not grouped by circles are nonlinearities for synchronous systems. The inclusions of CCI and ICI have led to higher saturation levels and slower saturation speeds (as a function of $R / d$ ). When only CCI is considered, we set $B=0$ in (21), (23), and (24a).

channel conditions. Because $R_{\text {sat }}$ defined above is often difficult to locate, as in [6], we choose $R_{\text {sat }}^{\prime} \stackrel{\text { def }}{=} 100 d$, where $d \stackrel{\text { def }}{=}\left(E_{c} / N_{0}\right)^{-1}$, as the reference point and redefine $R_{1 / 2}$ as the value such that $g_{\mathrm{opt}}\left(R_{1 / 2}\right)=0.5 g_{\mathrm{opt}}\left(R_{\mathrm{sat}}^{\prime}\right)$.

Define the average bit signal-to-noise ratio (SNR) $\gamma_{b}$ as $\gamma_{b}=$ $E\left(\alpha^{2}\right) E_{b} / N_{0}$, where $\alpha$ is a Rician or Rayleigh random variable characterizing the fading of the channel. The normalized nonlinearity $g(R) / d$ as a function of the normalized energy detector output $R / d$ for different number of interferers $J$ is depicted in Fig. 4. The nonlinearities of the chip-synchronous systems in Rayleigh [2] or Rician fading [6] have similar shapes but (25) renders different slopes and saturation levels; the difference is more evident when $J$ is large. The saturation levels of the nonlinearity over Rayleigh fading $(\Gamma=0)$ channels are much lower than that over Rician fading channels but the corresponding convergence (to the saturation level) speeds are faster. The influence of CCI and ICI is also indicated in the same figure. As we take into account more interference [larger $B$ in (21), (23), and (24a)] the saturation level tends to increase and the corresponding convergence speed decreases; see also the caption of Fig. 4.

Similar to the optimal normalized nonlinearity for synchronous systems [6], $g(x)$ can be well approximated by a soft-limiter. As mentioned before, the normalized nonlinearity has a slope close to one in its "more linear" region (see Fig. 4). We can then further approximate the soft-limiter by a $q$-level quantizer, $\tilde{g}(\cdot)$, with a uniform step size of $\nu=\Upsilon /(q-1) \stackrel{\text { def }}{=} g\left(R_{\text {sat }}^{\prime}\right) /(q-1)$, where $\Upsilon$ is the upper threshold of the quantizer. Such an approximation renders a larger quantization error for large normalized input $R / d$. A properly designed nonuniform quantizer will have a much smaller quantization error. However, we have compared the performance of both types of quantizers and found no significant difference in bit error probability performance. Hence, we will only consider uniform quantizers. The probability 
mass function (pmf) [15] of a uniform quantizer's outputs $\left\{Z_{n l}=\tilde{g}\left(R_{n l}\right)\right\}$ can be expressed as

$$
\begin{aligned}
P_{Z_{n l}}(z) & =\sum_{k=0}^{q-1} P_{r}\left(Z_{n l}=k \nu\right) \delta(z-k \nu) \\
\stackrel{\text { def }}{=} & \sum_{k=0}^{q-1} V_{n l}(k) \delta(z-k \nu)
\end{aligned}
$$

where the probabilities $V_{n l}(k)=P_{r}\left(Z_{n l}=k \nu\right)=P_{r}[k \nu \leq$ $R_{n l}<(k+1) \nu$ ] are given by [see (20)]

$$
V_{n l}(k)=\left\{\begin{aligned}
F_{R_{n l}}((k+1) \nu \mid m)-F_{R_{n l}}(k \nu \mid m), \\
k=0,1, \ldots, q-2, \\
1-F_{R_{n l}}(\Upsilon \mid m), \quad k=q-1
\end{aligned}\right.
$$

assuming that the $m$ th bin has been transmitted.

Designating $C_{n l}(k)$ as the probability mass

$$
C_{n l}(k)=P_{r}\{\text { the } n \text {th bin output }=k \nu, \text { when the number }
$$

of diversity branches used for combining is

$l+1 \mid$ the $m$ th bin is transmitted $\}$

we have

$$
C_{n(L-1)}(k)=P_{r}\left[Z_{n}=k \nu \mid \text { the } m \text { th bin is transmitted }\right]
$$

where $Z_{n}=\sum_{l=0}^{L-1} Z_{n l}$ is the output of an $L$-diversity combiner. The conditional pmf of $Z_{n}$ is given by

$$
P_{Z_{n}}(z)=\sum_{k=0}^{L(q-1)} C_{n(L-1)}(k) \delta(z-k \nu) .
$$

The associated probability mass $C_{n(L-1)}(k)$ can be computed recursively by [6]

$$
C_{n(L-1)}(k)=\sum_{w=w_{1}}^{w_{2}} C_{n(L-2)}(k-w) V_{n(L-1)}(w)
$$

where $w_{1}=\max [0, k-(L-1)(q-1)], w_{2}=\min (k, q-1)$, $C_{n 0}(k)=V_{n 0}(k)$.

With the aid of the above equations and the assumption that $\left\{R_{n l}\right\}$ are approximately i.i.d. r.v.s [3]-[6], the symbol error probability can be expressed as [6]

$$
\begin{aligned}
P_{S}(M ; L)= & 1-P_{r}[\text { correct symbol decision }] \\
= & 1-\sum_{k=1}^{L(q-1)} C_{m(L-1)}(k) \sum_{i=0}^{M-1}\left(\begin{array}{c}
M-1 \\
i
\end{array}\right) \\
& \cdot \frac{\left[C_{n(L-1)}(k)\right]^{i}}{i+1}\left[\sum_{l=0}^{k-1} C_{n(L-1)}(l)\right]^{M-i-1} \\
& +\frac{C_{m(L-1)}(0)}{M}\left[C_{n(L-1)}(0)\right]^{M-1} \cdot
\end{aligned}
$$

\section{OPTIMAL FH PATTERN SySTEM}

A set of optimal addresses that guarantee minimum CCI has been proposed by Solomon [7] and Einarsson [8]. In this section, two important properties of the optimal FH addresses are used in order to derive (34) and (35). These properties are: a) the cross correlation (pair-wise agreement) between any two users' addresses is at most one, i.e., any two different users' carriers hop to the same frequency at most once within a symbol period ( $L$ hops) and b) no address uses the same frequency twice within one symbol period.

\section{A. Performance Analysis}

Let $h_{l}, l=0,1, \ldots, L-1$, be the number of interfering tones that coincide with the lth diversity of a given row in the desired receiver's T-F matrix and define $h_{-1}=0$, $H_{l} \stackrel{\text { def }}{=} \sum_{k=0}^{l} h_{k}$. The cross-correlation property of the optimal FH pattern implies that $\sum_{i=0}^{L-1} h_{i} \leq J$, where $J$, as defined before, is the number of interferers. Property a) indicates that once $H_{l}$ interfering tones (that come from $H_{l}$ different users) have coincided with the desired user's pattern in the first $l$ hops, there can be at most $J-H_{l}$ interfering tones at the $(l+1)$ th hop. Property b) implies that at the $l$ th hop (starting at the zeroth hop) a user can only choose one out of the remaining $M-l$ frequencies available. The assumptions that $0 \leq \epsilon \leq 1$ and information symbols are i.i.d. tell us that each of these remaining candidate frequencies are to be equally likely selected with probability $1 /(M-l)$. Hence the probability of the interference pattern $\left\{h_{l}\right\}$ is given by

$$
\begin{aligned}
\operatorname{Pr} & \left(h_{0}, h_{1}, \ldots, h_{L-1}\right) \\
& =\prod_{l=0}^{L-1} \operatorname{Pr}\left(h_{l} \mid h_{0}, h_{1}, \ldots, h_{l-1}\right) \\
& =\prod_{l=0}^{L-1}\left(\begin{array}{c}
J-H_{l-1} \\
h_{l}
\end{array}\right)\left(\frac{1}{M-l}\right)^{h_{l}}\left(1-\frac{1}{M-l}\right)^{J-H_{l}}
\end{aligned}
$$

where $\operatorname{Pr}\left(h_{l} \mid h_{0}, h_{1}, \ldots, h_{l-1}\right)$ is the conditional probability that there are $h_{l}$ interfering tones at the $l$ th diversity branch given that $h_{k}$ tones hit the $k$ th diversity branch, $k=0,1, \ldots, l-1$. In Appendix B, we show that (34) can be simplified to

$$
\begin{aligned}
\operatorname{Pr}\left(h_{0}, h_{1}, \ldots, h_{L-1}\right) \\
=\left(\begin{array}{c}
J \\
h_{0}
\end{array}\right)\left(\begin{array}{c}
J-H_{0} \\
h_{1}
\end{array}\right) \cdots\left(\begin{array}{c}
J-H_{L-2} \\
h_{L-1}
\end{array}\right)\left(\frac{1}{M}\right)^{H_{L-1}} \\
\\
\cdot\left(1-\frac{L}{M}\right)^{J-H_{L-1}} .
\end{aligned}
$$

For a hard-limited combiner with a common threshold $\nu$ [3], the probabilities of having $l$ entries detected on the desired (information-bearing) row and an arbitrary undesired (noise-only) row, denoted by $P_{c}(l)$ and $P_{\bar{c}}(l)$, are given by

$$
\begin{aligned}
P_{c}(l)= & \sum_{E(l)} \operatorname{Pr}\left(h_{0}, h_{1}, \ldots, h_{L-1}\right) \\
& \cdot \prod_{k=0}^{L-1}\left[d_{k}+(-1)^{d_{k}} F_{R_{m k}}\left(\nu \mid m, h_{k}\right)\right] \\
P_{\bar{c}}(l)= & \sum_{E(l)} \operatorname{Pr}\left(h_{0}, h_{1}, \ldots, h_{L-1}\right) \\
& \cdot \prod_{k=0}^{L-1}\left[d_{k}+(-1)^{d_{k}} F_{R_{n k}}\left(\nu \mid m, h_{k}\right)\right]
\end{aligned}
$$


respectively, where $n \neq m$, and

$$
\begin{aligned}
& F_{R_{i k}}\left(r \mid m, h_{k}\right) \\
& \stackrel{\text { def }}{=} \operatorname{Pr}\left[R_{i k} \leq r \mid \text { the } m\right. \text { th bin is the message bin and the } \\
& i \text { th energy detector is hit by } h_{k} \text { interfering tones } \\
& \text { during the } k \text { th hop]. }
\end{aligned}
$$

The event $E(l)$ is the set $\left\{\left(d_{0}, d_{1}, \ldots, d_{L-1}, h_{0}, h_{1} \cdots h_{L-1}\right): \sum_{k=0}^{L-1} d_{k}=l\right.$, $\left.0 \leq \sum_{k=0}^{L-1} h_{k} \leq J\right\}$, where $d_{k}=0$ or 1 , depending on whether a miss or a detection occurs. As $P_{c}(l)=C_{m(L-1)}(l)$ and $P_{\bar{c}}(l)=C_{n(L-1)}(l)$, we can easily compute the associated bit (or symbol) error rate by substituting $q=2$, (36a) and (36b), into (33).

Note that the product terms in the right side of (36a) and (36b) represent the conditional probabilities that the combiner output at the message bin and a noise bin are $l$ given the interference pattern $\left\{h_{l}\right\}_{l=0}^{L-1}$. Therefore, to evaluate the performance of a synchronous soft-limited combiner we should replace these conditional probabilities by

$$
\prod_{k=0}^{L-1} V_{m k}\left(d_{k}\right), \quad \text { and } \prod_{k=0}^{L-1} V_{n k}\left(d_{k}\right), \quad n \neq m
$$

where the pmf of the soft-limited (quantized) output $V_{m k}\left(d_{k}\right)$, $d_{k}=0,1, \ldots, q-1$, is defined by (28) but the corresponding cdf is now given by

$$
\begin{aligned}
F_{R_{k l}}\left(R \mid m, h_{l}\right) & \\
= & \sqrt{R} \int_{0}^{\infty} J_{1}(\sqrt{R} \lambda) J_{0}\left(a \lambda \delta_{k m}\right) J_{0}^{h_{l}}(a \lambda) \\
& \cdot e^{-\lambda^{2} \sigma_{f}^{2}\left(\delta_{k m}+h_{l}\right) / 2} e^{-\lambda^{2} \sigma_{0}^{2} / 2} d \lambda, \quad k=m \text { or } n .
\end{aligned}
$$

\section{B. Reference Clock and Guard Time}

As has been mentioned, in a chip-asynchronous system, different received MFSK tones are no longer orthogonal and the nonorthogonality results in ICI, as shown in (13) and (A2). This section examines two methods for alleviating ICI and, to a lesser extent, CCI. The first approach is designed to minimize the timing offset among system users. This can be accomplished if the base station broadcasts a reference clock signal with which all the mobile units' local clocks will synchronize. Since all mobile units' symbol timing are synchronous with the reference, the timing misalignments among the received signals are only caused by the round-trip delays between mobile units and the base station. Such an arrangement is equivalent to the most primitive master-slave synchronization technique [16] that is usually used as part of the time distribution subsystem of a time-division multiple access (TDMA) network.

Given a data rate and the corresponding performance requirement, one can always find an appropriate cell size and diversity order that guarantee a maximum timing offset less than one chip duration. Let $\epsilon_{\max }<1$ be the maximum normalized timing offset and assume that $\epsilon_{j}$ s are i.i.d. random variables uniformly distributed within $\left[0, \epsilon_{\max }\right]$. The corresponding characteristic function, $\Phi_{X_{n l}}(\lambda \mid m)$, associated with the energy detector bank's output becomes

$$
\begin{aligned}
& \Phi_{X_{n l}}(\lambda \mid m) \\
&=e^{-\lambda^{2} \sigma_{0}^{2} / 2} e^{-\lambda^{2} \sigma_{f}^{2} \delta_{n m} / 2} J_{0}\left(a \lambda \delta_{n m}\right) \cdot \prod_{\rho=-B}^{B} \\
& \cdot\left\{(1-\mu)^{2}+\mu(1-\mu)\left[H_{1}(\lambda, \rho)+H_{2}(\lambda, \rho)\right]\right. \\
&\left.\quad+\mu^{2} G_{0}(\lambda, \rho)\right\}^{J}
\end{aligned}
$$

where

$$
\begin{aligned}
H_{1}(\lambda, \rho)= & \frac{1}{\epsilon_{\max }} \int_{0}^{\epsilon_{\max }} e^{-\epsilon^{2} \operatorname{sinc}^{2}(\rho \epsilon) \lambda^{2} \sigma_{f}^{2} / 2} \\
& \cdot J_{0}[a \epsilon \operatorname{sinc}(\rho \epsilon) \lambda] d \epsilon \\
H_{2}(\lambda, \rho)= & \frac{1}{\epsilon_{\max }} \int_{0}^{\epsilon_{\max }} e^{-(1-\epsilon)^{2} \operatorname{sinc}^{2}[\rho(1-\epsilon)] \lambda^{2} \sigma_{f}^{2} / 2} \\
& \cdot J_{0}[a(1-\epsilon) \operatorname{sinc}(\rho(1-\epsilon)) \lambda] d \epsilon \\
G_{0}(\lambda, \rho)= & \frac{1}{\epsilon_{\max }} \int_{0}^{\epsilon_{\max }} J_{0}[a(1-\epsilon) \operatorname{sinc}(\rho(1-\epsilon)) \lambda] \\
& \cdot e^{-\left\{[1-\epsilon]^{2} \operatorname{sinc}^{2}[\rho(1-\epsilon)]+\epsilon^{2} \operatorname{sinc}^{2}(\rho \epsilon)\right\} \lambda^{2} \sigma_{f}^{2} / 2} \\
& \cdot J_{0}[a \epsilon \operatorname{sinc}(\rho \epsilon) \lambda] d \epsilon .
\end{aligned}
$$

The above equations are similar to (21)-(22b), except that the timing offset $\epsilon$ is now uniformly distributed within the interval $\left(0, \epsilon_{\max }\right]$ instead of $(0,1]$.

The second approach achieves a similar result by inserting a guard time $T_{g}$ between two neighboring chips so that the chip duration is increased to $T_{c}^{\prime}=T_{c}+T_{g}$. However, in order to maintain the same data rate $R_{b}$ the diversity order has to be reduced according to

$$
L^{\prime}=\left\lfloor\frac{T_{s}}{T_{c}^{\prime}}\right\rfloor=\left\lfloor\frac{k / R_{b}}{T_{c}+T_{g}}\right\rfloor
$$

Suppose a reference clock is available and a guard time is inserted such that $T_{g} / T_{c}$ equals $\epsilon_{\max }$, then, during any given chip interval, the $j$ th interferer with a random hopping pattern and a nonzero normalized timing offset $\epsilon_{j}$ can hit the desired tone for either $\epsilon_{j}$ or $1-\epsilon_{j}$ of the time. Hence, without loss of generality, the interference model (17) becomes

$$
\begin{aligned}
I_{j n l}= & \sum_{k=0}^{M-1} \alpha_{j l} c_{j k l} e^{i \phi_{j l}}\left(1-\epsilon_{j}\right) \operatorname{sinc}\left[g_{k \ln }\left(1-\epsilon_{j}\right)\right] \\
& \cdot e^{i \pi g_{k: \ln }\left(1-\epsilon_{j}\right)}
\end{aligned}
$$

where $\eta=1$ is assumed. Note that there exists a tradeoff between the diversity order and the guard time length if a fixed data rate is to be maintained. Following an analogy procedure for deriving (21), we obtain

$$
\begin{array}{r}
\Phi_{X_{n l}}(\lambda \mid m)=e^{-\lambda^{2} \sigma_{0}^{2} / 2} e^{-\lambda^{2} \sigma_{f}^{2} \delta_{n m} / 2} J_{0}\left(a \lambda \delta_{n m}\right) \\
\cdot \prod_{\rho=-B}^{B}\left[1-\mu+\mu H_{0}(\lambda, \rho)\right]^{J}
\end{array}
$$


where

$$
\begin{aligned}
H_{0}(\lambda, \rho)= & \frac{1}{\epsilon_{\max }} \int_{0}^{\epsilon_{\max }} e^{-(1-\epsilon)^{2} \operatorname{sinc}^{2}[\rho(1-\epsilon)] \lambda^{2} \sigma_{f}^{2} / 2} \\
& \cdot J_{0}(a[1-\epsilon] \operatorname{sinc}[\rho(1-\epsilon)] \lambda) d \epsilon .
\end{aligned}
$$

The above equations can be used to derive the corresponding optimal or suboptimal nonlinearity.

\section{Two-Stage Detection}

\section{A. Principle of Two-Stage Detector}

Using the algebraic properties associated with the optimal FH pattern, Timor [9] proposed a multistage decoding method to cancel CCI in a noise-free environment. He noticed that the modulo arithmetic operation of two Reed-Solomon codewords is still a Reed-Solomon codeword, i.e., the interferer's FH pattern after dehopping is also a legitimate hopping pattern (codeword). Hence, after dehopping and hard-limiting, there are $J$ codewords and one complete signal row in the desired user's T-F matrix, if thermal noise is negligible. An error occurs when the $J$ codewords constitute one or more complete rows. The algorithm suggested in [9] provides a systematic procedure to test whether a complete row is the result of (at least) $L$ interferers (since no two users will coincide in at more than one chip). In the absence of thermal noise, the first stage (majority vote) detector always results in at least one complete row. If there are more than one complete rows, the detector enters the second stage using Timor's chip-by-chip test. However, the second stage cannot resolve the ambiguity completely. The probability of the residual ambiguity can be further reduced by employing multistage decoders having higher complexity [10].

\section{B. A Novel Two-Stage Detector}

The multistage detector suggested by Timor [9] assumed a hard-decision diversity combining rule and a zero thermal noise environment. When the effect of thermal noise is taken into consideration, the probability of both chip deletion (a message chip fails to pass the hard limiter threshold) and chip insertion (a nonmessage chip passes the hard limiter threshold) will not be zero. It is likely that the message chips do not form a complete row and the interference test is incapable of detecting a false complete row. To deal with such a practical situation we propose the following two-stage detector. The first stage locates the row (in the T-F matrix) with the largest hard-limited combining output. If there are $I(\geq 2)$ rows having the same largest output value, the detector then enters the second stage where it:

1) performs an interference test on each chip in the $i$ th largest row and records the number of chips that pasess the test, say $p_{i}$. A chip that passes the interference test is assumed to have been corrupted by an interferer.

2) repeats the above step for $i=1,2, \ldots, I$.

3 ) finds the row that yields the smallest number of passed chips, say $p_{m}$, and decodes $m$ as the correct message.

4) determines if there are two or more smallest $p_{i}$ s and chooses one at random as the message.
The so-called interference test mentioned in Step 1) is designed to test whether a particular chip has CCI from other users. In other words, the test checks if any interferer might use a particular frequency in a given chip interval.

When soft-limited combining is used in the first stage, we select those rows with the largest combining output value say $R_{\max }$ plus those whose combined output values are greater than $R_{\max }-D$ but less than $R_{\max }$ to enter the second stage. The threshold value $D$ can be optimized to yield a minimum error probability. We further divide the $I$ selected rows into two categories - the first category, $G_{1}$, are those that have the largest output while the rest, $G_{2}$, belong to the second category. In other words, $R_{\max } \stackrel{\text { def }}{=} \max _{n} Z_{n}, G_{1}=\left\{k \mid Z_{k}=R_{\max }\right\}$ and $G_{2}=\left\{k \mid R_{\max }>Z_{k}>R_{\max }-D\right\}$. Step 4) is then modified as follows.

$4^{\prime}$ ) If there are two or more smallest $p_{i}$ s and the associated rows belong to both categories, choose one out of $G_{1}$ at random and decode it as the message.

The interference test proposed in [9] is designed for use with a special class of optimal hopping patterns. This class is not suitable for asynchronous operations. Appendix $\mathrm{C}$ shows that for the class of the optimal asynchronous hopping patterns [8] the interference test can still be used if the initial realignment procedure is properly modified.

Multistage detectors that consider all possible combinations of users' addresses and message patterns were discussed in [10], [11]. Such a complete (multistage) detection scheme is very complicated: instead of $L+L(L-2)$ cycle-shifting operations for resolving an ambiguous row, a complete decoding requires $L\left(2^{k}-1\right)$ cycle-shifting operations. Since $L \ll 2^{k}=M$, the two-stage detector has a much smaller complexity than that of a complete detector.

\section{NUMERICAL RESULTS AND DISCUSSIONS}

Unless otherwise specified, the following system parameters are used: $W=20 \mathrm{MHz}, R_{b}=32.895 \mathrm{kHz}, M=256, L=19$, and $\eta=1$.

To examine the effect of ICI we consider $2 B=20$ adjacent channels around the desired channel since no noticeable capacity degradation is found if $B$ is greater than 10; see Fig. 5. In other words, ICI caused by an interferer whose carrier is $10 \Delta f$ $\mathrm{Hz}$ away from the desired tone is negligible. System capacity degradation caused by ICI and CCI can be found in Fig. 6 where we have depicted the BER performance as a function of the number of active users. The two CCI curves are obtained by ignoring ICI $(B=0)$. The system performance of both hard-limited and soft-limited combining are also demonstrated in Fig. 6 from which we find that at a BER below $10^{-3}$, single-stage soft-limited receivers can accommodate 10 to 26 more users (or $1.6 \%$ to $4.3 \%$ higher spectral efficiency) than their hard-limited counterparts. The threshold $\Upsilon \triangleq 0.5 g\left(R_{\text {sat }}^{\prime}\right)$ is used for hard-limited combining.

The influence of channel spacing $\Delta f$ is illustrated in Fig. 7 . Using the definition of spectral efficiency (3), one can easily come to the conclusion that the system with $\eta=1$ is the best choice in terms of spectral efficiency. Fig. 8 demonstrates the 


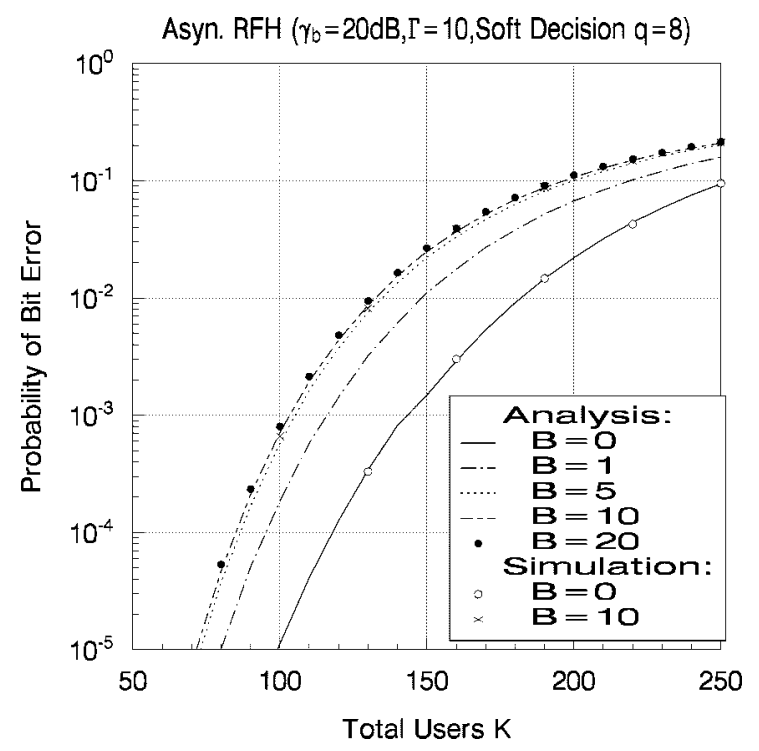

Fig. 5. Effect of ICI on the BER performance of the asynchronous FHMA/ MFSK system, where $2 B$ is the number of adjacent channels considered and $q=8$ quantization levels along with random frequency hopping (RFH) patterns are assumed.

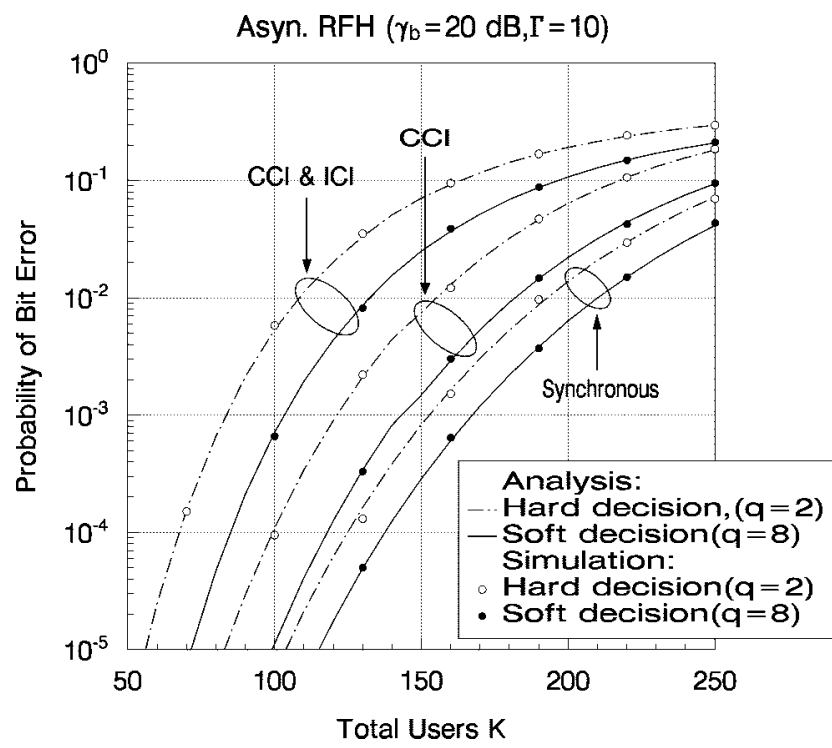

Fig. 6. Performance comparison: hard versus soft decisions and the influence of ICI and CCI; ICI is not included $(B=0)$ in obtaining those two curves labeled by CCI; $q$ is the number of quantization levels of the soft-limiter.

effect of using a reference clock to minimize the maximum normalized timing offset among users to $\epsilon_{\max }$. Obviously, the smaller $\epsilon_{\max }$ is (see those two curves with $T_{g}=0$ ), the better the BER performance becomes. If guard time $T_{g}=\epsilon_{\max }$ is inserted, further improvement can be obtained, as also demonstrated in Fig. 8. For a system with $M=256$ and $T_{g}=0.1 T_{c}$ or $T_{g}=0.2 T_{c}$, (41) implies that the diversity order $L$ has to be reduced to 17 or 16 . The effect of reference clock and guard time insertion in mitigating ICI is obvious but that in lessening CCI is not so obvious. The "net" effect of CCI is assessed by evaluating the system performance without taking ICI into account $(B=0)$. Fig. 9 shows that CCI can indeed be reduced if proper design parameters are selected. It also indicates that systems using both mitigation schemes do necessarily yield per-

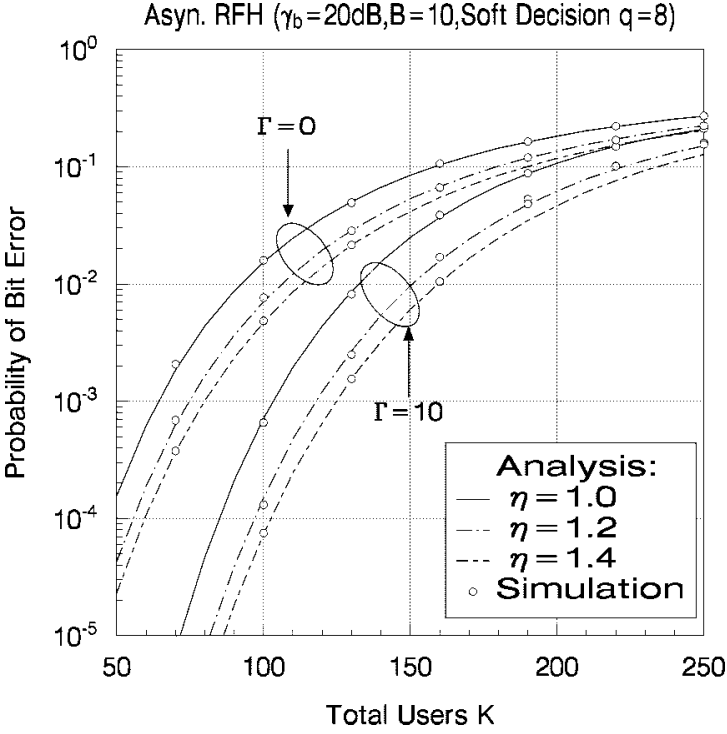

Fig. 7. The impact of normalized channel spacing on the BER performance of asynchronous FHMA/MFSK systems.

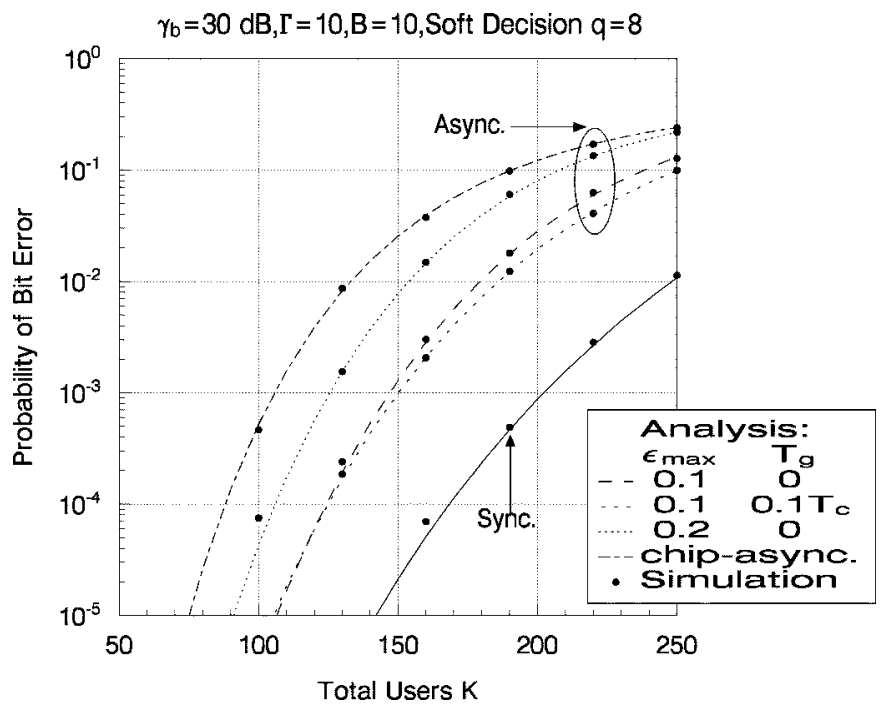

Fig. 8. BER performance improvements for chip-asynchronous systems when a reference clock is used or/and a guard time is inserted. The maximum chipduration normalized timing offset is denoted by $\epsilon_{\max }$. The performance of the synchronous system (the right-most curve and dots) is provided for comparison purposes.

formance better than those using only a pilot clock, for, as just mentioned, inserting a guard-time is likely to force us to reduce the diversity order $L$ in order to maintain the same data rate.

Figs. 5-9 assume random hopping patterns are used by the system. The effectiveness of the proposed two-stage detector is shown in Fig. 10. Comparing Figs. 8 and 10, we find that the use of an optimal pattern brings about little improvement. This is due to the fact that the probability of more than one collision during a symbol period is very small for the system parameters under consideration ( $M=256, L=19$ or 11). It is noticed that the improvement over the single-stage detector is most impressive when the system is in the symbol-synchronous mode. Obviously, the algebraic properties of the hopping pattern that we have used in designing the interference test is not very useful for chip-asynchronous interference. 


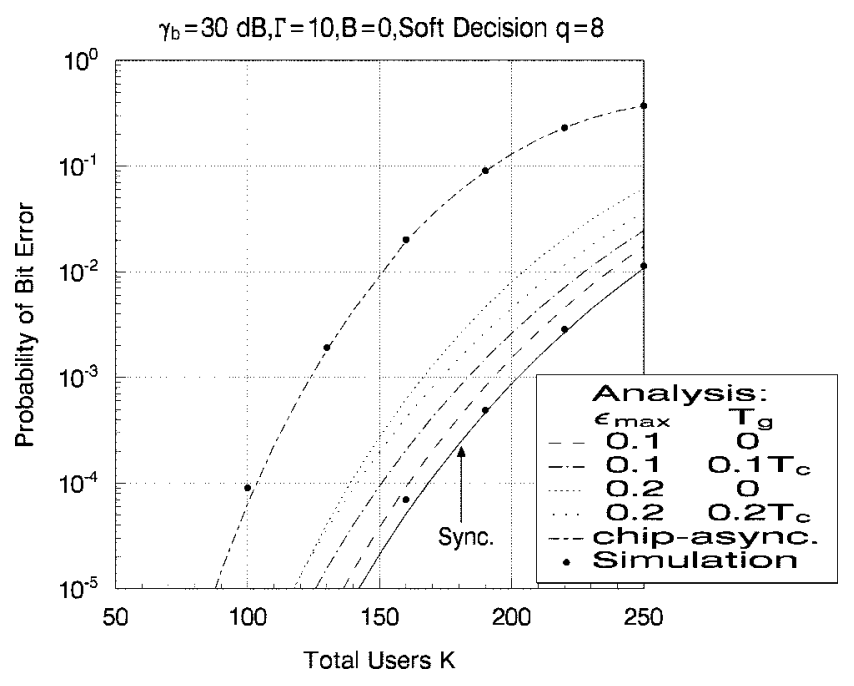

Fig. 9. Effects of reference clock or/and guard time on CCI. These curves were obtained by ignoring ICI $(B=0)$. The left-most curve and dots correspond to the performance of the chip-asynchronous system without these two remedies.

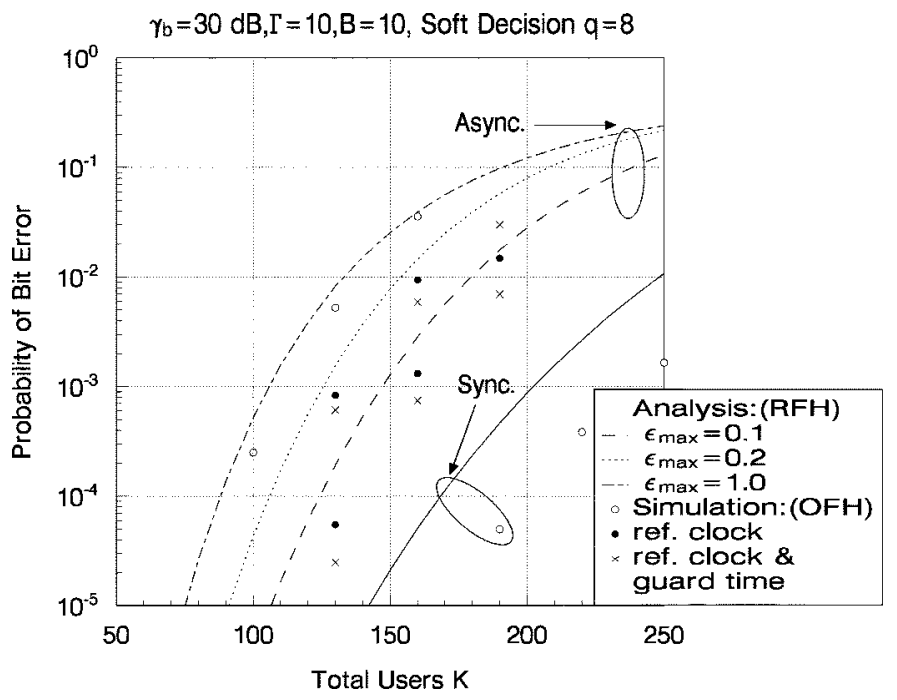

Fig. 10. Effect of the second stage detection: optimal frequency-hopping $(\mathrm{OFH})$ pattern set is used and random frequency-hopping $(\mathrm{RFH})$ patterns are used.

From the above error probability curves we can derive the ultimate system performance measure-system capacity $K_{\max }$ and spectral efficiency $\beta$. We summarize some of the selected $\left(K_{\max }, \beta\right)$ values at $\mathrm{BER}=10^{-3}$ along with the corresponding system parameters in Table I. The performance of chip-asynchronous systems is much inferior to that of synchronous systems, especially in high $\gamma_{b}$ s. For example, Table I indicates that at $\mathrm{BER}=10^{-3}$ the latter allow 64 more users when $\gamma_{b}=20$ $\mathrm{dB}, \Gamma=10$ and 97 more users when $\gamma_{b}=30 \mathrm{~dB}, \Gamma=10$. The values in parentheses are obtained when the system inserts a proper guard time; see (41)-(44). Similar to the conclusion we derived from Fig. 10, the advantage of using the optimal hopping patterns is most obvious in synchronous systems in which the two-stage receiver obtains a $10 \%$ to $20 \%$ increase in system capacity. However, when the system becomes asynchronous this benefit is drastically reduced and, as $\epsilon_{\max }$ increases, it gradually disappears.
TABLE I

SYSTEM CAPACITY $K_{\text {max }}$, SPECTRAL EFFICIENCY $\beta$, AND THE ASSOCIATED SYSTEM PARAMETERS

\begin{tabular}{|c|c|c|c|c|c|c|}
\hline FH pattern & Sync & $\epsilon_{\max }$ & $\Gamma$ & $\gamma_{b}$ & $K_{\max }$ & $\beta \%$ \\
\hline \multirow{16}{*}{ random } & \multirow{4}{*}{ yes } & \multirow{4}{*}{ - } & 0 & 20 & 135 & 22.2 \\
\hline & & & 0 & 30 & 195 & 32.1 \\
\hline & & & 10 & 20 & 167 & 27.5 \\
\hline & & & 10 & 30 & 203 & 33.4 \\
\hline & \multirow{12}{*}{ no } & \multirow{4}{*}{0.1} & 0 & 20 & $100(103)^{*}$ & $16.4(16.9)$ \\
\hline & & & 0 & 30 & $115(124)$ & $18.9(20.4)$ \\
\hline & & & 10 & 20 & $137(138)$ & $22.5(22.7)$ \\
\hline & & & 10 & 30 & $147(150)$ & $24.2(24.7)$ \\
\hline & & \multirow{4}{*}{0.2} & 0 & 20 & $83(86)$ & $13.7(14.1)$ \\
\hline & & & 0 & 30 & $91(96)$ & $15.0(15.8)$ \\
\hline & & & 10 & 20 & $120(121)$ & $19.7(19.9)$ \\
\hline & & & 10 & 30 & $126(128)$ & $20.7(21.1)$ \\
\hline & & \multirow{4}{*}{1.0} & 0 & 20 & 64 & 10.5 \\
\hline & & & 0 & 30 & 66 & 10.8 \\
\hline & & & 10 & 20 & 103 & 16.9 \\
\hline & & & 10 & 30 & 106 & 17.4 \\
\hline \multirow{16}{*}{$\begin{array}{c}\text { optimal } \\
\text { (2-stages) }\end{array}$} & \multirow{4}{*}{ yes } & & 0 & 20 & 151 & 24.8 \\
\hline & & & 0 & 30 & 225 & 37.0 \\
\hline & & & 10 & 20 & 185 & 30.4 \\
\hline & & & 10 & 30 & 240 & 39.5 \\
\hline & \multirow{12}{*}{ no } & \multirow{4}{*}{0.1} & 0 & 20 & $111(116)$ & $18.3(19.1)$ \\
\hline & & & 0 & 30 & $126(135)$ & $20.7(22.2)$ \\
\hline & & & 10 & 20 & $149(151)$ & $24.5(24.8)$ \\
\hline & & & 10 & 30 & $157(164)$ & $25.8(27.0)$ \\
\hline & & \multirow{4}{*}{0.2} & 0 & 20 & $91(96)$ & $15.0(15.8)$ \\
\hline & & & 0 & 30 & $99(106)$ & $16.3(17.4)$ \\
\hline & & & 10 & 20 & $129(131)$ & $21.2(21.5)$ \\
\hline & & & 10 & 30 & $132(137)$ & $21.7(22.5)$ \\
\hline & & \multirow{4}{*}{1.0} & 0 & 20 & 67 & 11.0 \\
\hline & & & 0 & 30 & 68 & 11.1 \\
\hline & & & 10 & 20 & 109 & 17.9 \\
\hline & & & 10 & 30 & 112 & 18.4 \\
\hline
\end{tabular}

\section{CONCLUSION}

This paper deals with noncoherent detection of fast FHMA/ MFSK signals in an environment characterized by frequencynonselective Rician fading. We derived a nearly optimal nonlinearity for diversity combining in the asynchronous mode. A suboptimal realization using quantizers (soft limiters) was proposed and analyzed. For other operation scenarios (limited time offsets, guard time insertion) considered we also derived the corresponding characteristic functions that are needed to obtain the associated optimal nonlinearities.

We investigated the impact of the FH patterns used and found that, for single-stage detectors, the use of optimal FH patterns yields little BER reduction when compared with systems using random FH patterns. On the other hand, the algebraic properties of the optimal pattern renders itself suitable for multistage detection. We suggested a two-stage detector which was shown to be effective in the symbol-synchronous mode for combating both ICI and CCI. Users' clock misalignment destroys the signal orthogonality and makes the algebraic properties of the optimal hopping pattern less useful, which resulted in a significant performance degradation for both single- and two-stage detectors. Two methods for lessening the performance degradation and containing the interference were thus examined. Numerical results proved that these methods provide a sufficient interference-reduction capability under the assumed channel condition. 


\section{APPENDIX A}

\section{DERIVATIONS OF (19)-(22b)}

The complex random variable $U_{n l}$, as can be seen from (15), is the sum of three independent complex random variables. For a fixed set of $\left\{\alpha_{m l}, \alpha_{j l}, c_{j k l}, \epsilon_{j}\right\}$, each of these r.v.s has a spherical symmetric probability distribution [13], i.e., the corresponding pdf is independent of the complex r.v.s direction (phase) and is a function of its magnitude only. It follows immediately from the independence assumption that the characteristic function of $X_{n l} \stackrel{\text { def }}{=}\left|U_{n l}\right|$ for given $\left\{\alpha_{m l}, \alpha_{j l}, c_{j k l}, \epsilon_{j}\right\}$, which is expressed as $\Phi_{X_{n l}}\left(\lambda \mid \alpha_{m l}, \alpha_{j l}, c_{j k l}, \epsilon_{j}\right)$, is the product of the conditional characteristic functions of $s_{n l}$, $I_{n l}=\sum_{j=1}^{J} I_{j n l}$ and $z_{n l}[\operatorname{see}(15)] ;$ i.e.,

$$
\Phi_{X_{n l}}\left(\lambda \mid \alpha_{m l}, \alpha_{j l}, c_{j k l}, \epsilon_{j}\right)=\Phi_{s}(\lambda) \Phi_{I}(\lambda) \Phi_{n}(\lambda)
$$

where, on the right-hand side of the equation, for simplicity, we express the conditional characteristic functions of $s_{n l}, I_{n l}$ and $z_{n l}$ as $\Phi_{s}(\lambda), \Phi_{I}(\lambda)$ and $\Phi_{n}(\lambda)$.

Assuming $\eta=1$, substituting (16) and (17) into (15), and restricting ICI to $\pm B$ adjacent channels, we have

$$
\begin{aligned}
& U_{n l}=\alpha_{m l} \delta_{n m} e^{i \phi_{m l}} \\
&+\sum_{j=1}^{J}\left[\sum_{\rho=-B}^{B} \alpha_{j l} c_{j(n+\rho) l} e^{i \phi_{j l}}\right. \\
& \cdot\left(1-\epsilon_{j}\right) \operatorname{sinc}\left[\rho\left(1-\epsilon_{j}\right)\right] e^{i\left[\pi \rho\left(l-\epsilon_{j}\right)\right]} \\
&+\sum_{\rho=-B}^{B} \alpha_{j(l+1)} \\
&\left.\quad \cdot c_{j(n+\rho)(l+1)} e^{i \phi_{j(l+1)}} \epsilon_{j} \operatorname{sinc}\left(\rho \epsilon_{j}\right) e^{i\left[\pi \rho\left(2-\epsilon_{j}\right)\right]}\right] \\
&+z_{n l} .
\end{aligned}
$$

Applying the theory of spherical symmetric random vectors [12], [13], we obtain

$$
\begin{aligned}
\Phi_{s}(\lambda)= & J_{0}\left(\alpha_{m l} \delta_{n m} \lambda\right) \\
\Phi_{I}(\lambda)= & \prod_{j=1}^{J} \prod_{\rho=-B}^{B} J_{0}\left(\alpha_{j(l+1)} c_{j(n+\rho)(l+1)} \epsilon_{j} \cdot \operatorname{sinc}\left[\rho \epsilon_{j}\right] \lambda\right) \\
& \cdot \prod_{j=1}^{J} \prod_{\rho=-B}^{B} J_{0}\left(\alpha_{j l} c_{j(n+\rho) l}\left(1-\epsilon_{j}\right) \operatorname{sinc}\left[\rho\left(1-\epsilon_{j}\right)\right] \lambda\right) \\
\Phi_{n}(\lambda)= & e^{-\sigma_{0}^{2} \lambda^{2} / 2}
\end{aligned}
$$

where $J_{0}(\cdot)$ is the zeroth order Bessel function of the first kind. As mentioned in Section II, for a given $(j, l)$ pair, $\left\{c_{j n l}\right\}$ are not independent and only one of them is nonzero. However, it has been shown in [3] and [6] that when $K \gg 1$ and the independent random hopping patterns are used, $\left\{c_{j n l}\right\}$ are approximately i.i.d. with a common pdf

$$
f_{c_{j n l}}(x)=(1-\mu) \delta(x)+\mu \delta(x-1)
$$

where $\mu=1 / M$. Taking expectation on $\Phi_{I}(\lambda)$ with respect to $c_{j n l}$ and substituting the resulting expression together with (A3) and (A5) into (A1), we obtain

$$
\begin{aligned}
& \Phi_{X_{n l}}\left(\lambda \mid m, \alpha_{m l}, \alpha_{j l}, \epsilon_{j}\right) \\
& \quad=e^{-\sigma_{0}^{2} \lambda^{2} / 2} J_{0}\left(\alpha_{m l} \delta_{n m} \lambda\right) \cdot \prod_{j=1}^{J} \prod_{\rho=-B}^{B} \Phi_{I}\left(\lambda \mid \alpha_{j l}, \epsilon_{j}, \rho\right)
\end{aligned}
$$

where

$$
\begin{aligned}
\Phi_{I}( & \left.\lambda \mid \alpha_{j l}, \epsilon_{j}, \rho\right) \\
= & \left\{1-\mu+\mu J_{0}\left(\alpha_{j(l+1)} \epsilon_{j} \cdot \operatorname{sinc}\left[\rho \epsilon_{j}\right] \lambda\right)\right\} \\
& \cdot\left\{1-\mu+\mu J_{0}\left(\alpha_{j l}\left(1-\epsilon_{j}\right) \operatorname{sinc}\left[\rho\left(1-\epsilon_{j}\right)\right] \lambda\right)\right\} .
\end{aligned}
$$

Averaging (A7) over $\alpha_{m l}$ and $\alpha_{j l}$ and using (6.633.4) of [17] we obtain

$$
\begin{aligned}
& \Phi_{X_{n l}}\left(\lambda \mid m, \epsilon_{j}\right) \\
& \quad=e^{-\sigma_{0}^{2} \lambda^{2} / 2} e^{-\lambda^{2} \sigma_{f}^{2} \delta_{r m} / 2} J_{0}\left(a \lambda \delta_{n m}\right) \cdot \prod_{j=1}^{J} \prod_{\rho=-B}^{B} \Phi_{I}\left(\lambda \mid \epsilon_{j}, \rho\right)
\end{aligned}
$$

where

$$
\begin{aligned}
\Phi_{I}\left(\lambda \mid \epsilon_{j}, \rho\right) \\
=\left\{1-\mu+\mu e^{-\epsilon_{j}^{2} \operatorname{sinc}^{2}\left(\rho \epsilon_{j}\right) \lambda^{2} \sigma_{f}^{2} / 2} \cdot J_{0}\left(a \epsilon_{j} \cdot \operatorname{sinc}\left[\rho \epsilon_{j}\right] \lambda\right)\right\} \\
\cdot\left\{1-\mu+\mu e^{-\left(1-\epsilon_{j}\right)^{2} \operatorname{sinc}^{2}\left[\rho\left(1-\epsilon_{j}\right)\right] \lambda^{2} \sigma_{f}^{2} / 2}\right. \\
\left.\cdot J_{0}\left(a\left(1-\epsilon_{j}\right) \operatorname{sinc}\left[\rho\left(1-\epsilon_{j}\right)\right] \lambda\right)\right\}
\end{aligned}
$$

but $\epsilon_{j}, j=1,2, \ldots, J$, are i.i.d. r.v.s with a common uniform distribution within $(0,1]$. By taking the average over $\epsilon_{j}$, we then obtain (21) and the ensuing two equations. As $X_{n l}$ is the length of a two-dimensional random vector $U_{n l}$ that has a spherical symmetric distribution, we can apply the Hankeltransform-like relations between $\Phi_{X_{n l}}(\lambda \mid m)$ and the pdf and cdf of $X_{n l}, f_{X_{n l}}(r \mid m), F_{X_{n l}}(r \mid m)[12,(31)]$

$$
\begin{aligned}
\Phi_{X_{n l}}(\rho \mid m) & =\int_{0}^{\infty} J_{0}(r \rho) f_{X_{n l}}(r \mid m) d r \\
f_{X_{n l}}(r \mid m) & =\int_{0}^{\infty} r \rho J_{0}(r \rho) \Phi_{X_{n l}}(\rho \mid m) d \rho \\
F_{X_{n l}}(r \mid m) & =r \int_{0}^{\infty} \rho J_{1}(r \rho) \Phi_{X_{n l}}(\rho \mid m) d \rho
\end{aligned}
$$

and the change of variables $R_{n l}=X_{n l}^{2}$ to obtain (19) and (20). The above equations, (A11)-(A13), or their variations can also be found in [18] and [19]. The pdf (cdf) derivations for the other cases, e.g., $\eta \neq 1$, are similar hence will not be repeated. 
APPENDIX B

DERIVATION OF (35)

Expanding the right-hand side of (34), we obtain

$$
\begin{aligned}
\operatorname{Pr}\left(h_{0}, h_{1}, \ldots, h_{L-1}\right) & \left(\begin{array}{c}
J \\
h_{0}
\end{array}\right)\left(\frac{1}{M}\right)^{h_{0}}\left(1-\frac{1}{M}\right)^{J-h_{0}}\left(\begin{array}{c}
J-h_{0} \\
h_{1}
\end{array}\right) \\
& \cdot\left(\frac{1}{M-1}\right)^{h_{1}}\left(1-\frac{1}{M-1}\right)^{J-h_{0}-h_{1}} \\
& \cdots\left(\begin{array}{c}
J-h_{0}-h_{1}-\cdots-h_{L-2} \\
h_{L-1}
\end{array}\right)\left(\frac{1}{M-L+1}\right)^{h_{L-1}} \\
& \cdot\left(1-\frac{1}{M-L+1}\right)^{J-h_{0}-h_{1}-\cdots-h_{L-1}} \cdot
\end{aligned}
$$

By rearranging (B1), we obtain

$$
\begin{aligned}
\operatorname{Pr}\left(h_{0}, h_{1}, \ldots, h_{L-1}\right) & \left(\begin{array}{c}
J \\
h_{0}
\end{array}\right)\left(\begin{array}{c}
J-h_{0} \\
h_{1}
\end{array}\right)\left(\begin{array}{c}
J-h_{0}-h_{1} \\
h_{2}
\end{array}\right) \\
& \ldots\left(\begin{array}{c}
\left.J-h_{0}-h_{1}-\cdots-h_{L-2}\right) \\
h_{L-1}
\end{array}\right. \\
& \cdot\left(\frac{1}{M}\right)^{h_{0}}\left(\frac{M-1}{M}\right)^{J-h_{0}}\left(\frac{1}{M-1}\right)^{h_{1}} \\
& \cdot\left(\frac{M-2}{M-1}\right)^{J-h_{0}-h_{1}} \cdots\left(\frac{1}{M-L+1}\right)^{h_{L-1}} \\
& \cdot\left(\frac{M-L}{M-L+1}\right)^{J-h_{0}-h_{1}-\cdots-h_{L-1}} \\
= & \left.\left(\begin{array}{c}
J \\
h_{0}
\end{array}\right)\left(\begin{array}{c}
J-H_{0} \\
h_{1}
\end{array}\right)^{J-H_{1}}\right)^{h_{2}}\left(\begin{array}{c}
J-H_{L-2} \\
h_{L-1}
\end{array}\right)^{J-H_{0}}(M-2)^{J-H_{1}} \\
& {\left[\left(\frac{1}{M}\right)^{J}(M-1)^{J-H_{0}}\left(\frac{1}{M-1}\right)^{(\mathrm{B} 2)}\right.} \\
& \quad \ldots\left(\frac{1}{M-L+1}\right)^{J-H_{L-2}}(M-L)^{J-H_{L-1}}
\end{aligned}
$$

It is easy to see that the above equation is equal to (35).

\section{APPENDIX C \\ AN INTERFERENCE TEST}

The interference test proposed by Timor [9] for use in a twostage decoding algorithm is valid for the address sets generated by (4). Timor did not deal with the other address assignment scheme presented in [8] that determines the transmitted frequency vector for the $j$ th user by

$$
\mathbf{f}_{j}=m_{j} \boldsymbol{\beta} \oplus \boldsymbol{\Lambda}_{\mathbf{j}}
$$

where $\boldsymbol{\beta}=\left(1, \beta, \beta^{2}, \ldots, \beta^{L-1}\right)$ and $\boldsymbol{\Lambda}_{j}=\left(\lambda_{j}, \lambda_{j}, \ldots, \lambda_{j}\right)$ is a constant $L$-tuple representing the address of the $j$ th user and $m_{j}$ is the message symbol (MFSK tone); both $\lambda_{j}$ and $m_{j}$ are elements of $G F(M)$. This address assignment is optimal for chip-asynchronous systems [8].

We want to demonstrate that a simple modification on the original interference test [9] is sufficient for use in a system that employs the FH patterns (C1). For such a system, the fre- quency-dehopper has to perform the inverse operation of (C1). Chip-by-chip detection would result in a complete $m_{j}$ th row in the dehopped T-F matrix $\mathbf{A}_{\mathbf{j}}$ of user $j$ if no noise is present. Suppose CCI results in another complete row $\mathbf{X}^{\prime}$ in $\mathbf{A}_{\mathbf{i}}$. The address assignment scheme assures that every chip in $\mathbf{X}^{\prime}$ must come from different users and therefore at least $L$ users $j_{1}, j_{2}, \ldots, j_{L}$ are needed to produce a complete row. The user that contributes to the $l$ th entry of $\mathbf{X}^{\prime}$ is assumed to be the $j_{l}$ th user. If there are more than one user that cause this hit, let $j_{l}$ be any one of them.

From this address assignment, the $k$ th entry of the $j_{i}$ th user in $\mathbf{A}_{\mathbf{i}}$ will be at row $r_{l}(k)$ and column $k$, where

$$
\begin{aligned}
r_{l}(k) & =\left(m_{j_{l}} \beta^{k-1}+\lambda_{j_{l}}-\lambda_{i}\right) \beta^{-(k-1)} \\
& =\delta_{l} \beta^{-(k-1)}+m_{j_{l}}, \quad k=1,2, \ldots, L
\end{aligned}
$$

and $\delta_{l} \triangleq \lambda_{j_{l}}-\lambda_{i}$. Note that $\delta_{l} \neq 0$ for all $j_{l}, l=1,2, \ldots, L$, and $r_{l}(l)$ is the row in which the entry of the $j_{l}$ th user appears at the $l$ th column. Since this row is $\mathbf{X}^{\prime}$ we obtain

$$
r_{l}(l)=\delta_{l} \beta^{-(l-1)}+m_{j_{l}}=X^{\prime} .
$$

Comparing the above equation with $[9,(5)]$, we conclude that the rest of the interference test procedure can be carried out in exactly the same way as that presented in [9] if we replace $\beta$ by $\beta^{-1}$.

\section{ACKNOWLEDGMENT}

The authors would like to thank the anonymous reviewers for their careful reading and valuable suggestions.

\section{REFERENCES}

[1] D. J. Goodman, P. S. Henry, and V. K. Prabhu, "Frequency-hopped multilevel FSK for mobile radio," Bell Syst. Tech. J., vol. 59, no. 7, pp. 1257-1275, Sept. 1980.

[2] O. C. Yue, "Maximum likelihood combining for noncoherent and differential coherent frequency-hopping multiple-access systems," IEEE Trans. Inform. Theory, vol. IT-28, pp. 631-639, July 1982.

[3] - "Performance of frequency-hopping multiple-access multilevel FSK systems with hard-limited and linear combining," IEEE Trans. Commun., vol. COM-29, pp. 1687-1694, Nov. 1981.

[4] U. C. Fiebig, "On the efficiency of fast frequency hopping multipleaccess systems," in ICC'92 Conf. Rec., July 1992, pp. 302.2.1-302.2.5.

[5] P. Yegani and C. D. McGillem, "FH-MFSK multiple-access communication systems performance in the factory environment," IEEE Trans. Veh. Technol., vol. 42, pp. 148-155, May 1993.

[6] C. P. Hung and Y. T. Su, "Diversity combining considerations for incoherent frequency hopping multiple access systems," IEEE J. Select. Areas Commun., vol. 13, pp. 333-344, Feb. 1995.

[7] G. Solomon, "Optimal frequency hopping sequences for multiple access," in Proc. 1973 Symp. Spread Spectrum Commun., San Diego, CA, March 13-16, 1973, pp. 33-35.

[8] G. Einarsson, "Address assignment for a time-frequency-coded, spreadspectrum system,” Bell Syst. Tech. J., vol. 59, no. 7, pp. 1241-1255, Sept. 1980.

[9] U. Timor, "Improved decoding scheme for frequency-hopped multilevel FSK system," Bell Syst. Tech. J., vol. 59, no. 10, pp. 1839-1855, Dec. 1980.

[10] - "Multistage decoding of frequency-hopped FSK system," Bell Syst. Tech. J., vol. 60, no. 4, pp. 471-483, Apr. 1981.

[11] T. Mabuchi, R. Kohno, and H. Imai, "Multiuser detection scheme based on cancelling cochannel interference for $M \mathrm{FSK} / \mathrm{FH}-\mathrm{SSMA}$ system," IEEE J. Select. Areas Commun., vol. 12, pp. 593-604, May 1994.

[12] R. D. Lord, "The use of Hankel transform in statistics," Biometrika, vol. 41, pp. 44-55, 1954.

[13] J. S. Bird and D. A. George, "The use of the Fourier-Bessel series in calculating error probabilities for digital communications systems," IEEE Trans. Commun., vol. COM-29, pp. 1357-1365, Sept. 1981.

[14] Y. T. Su, Y.-S. Shen, and C.-Y. Hsiao, "A study of frequency-hopping multiple access mobile satellite systems," National Chiao Tung Univ., Final Report, NSC84-2612-E009-003, Aug. 1995. 
[15] G. R. Grimmett and D. R. Stirzaker, Probability and Random Processes. New York: Oxford Univ. Press, 1992, ch. 3.

[16] W. C. Lindsey, F. Ghazvinian, W. C. Hagmann, and K. Dessouky, "Network synchronization," Proc. IEEE, vol. 73, Oct. 1985.

[17] I. S. Gradshteyn and I. M. Ryzhik, Table of Integrals, Series, and Products. New York: Academic, 1980.

[18] S. O. Rice, "Mathematical analysis of random noise," Bell Syst. Tech. J., vol. 24, pp. 46-156, 1945.

[19] K. S. Miller, Multidimensional Gaussian Distribution. New York: Wiley, 1964, pp. 66-69.

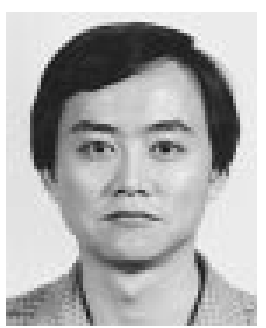

Yu T. Su (M'98) received the B.S.E.E. degree from Tatung Institute of Technology, Taipei, Taiwan, and the Ph.D. degree from the University of Southern California, in 1974 and 1983, respectively.

From 1983 to 1989 he was with LinCom Corporation, Los Angeles, CA, where he was involved in the design of various measurement and digital satellite communication systems. Since September 1989 he has been with the Department of Communication Engineering and Microelectronics and Information Systems Research Center, National Chiao Tung University, Hsinchu, Taiwan. His research interests include communication theory and statistical signal processing.

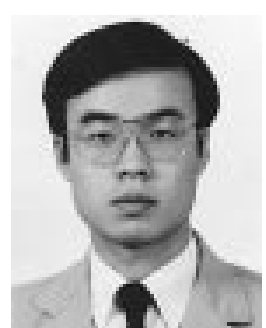

Ye-Shun Shen received the M.S. degree in communication engineering from the National Chiao Tung University, Hsinchu, Taiwan, in 1996. He is currently a Ph.D. candidate at the Institute of Electrical Engineering, National Cheng Kung University, Tainan, Taiwan.

Since 1993, he has been with Chunghwa Telecom, where he is a Senior Engineer. His research interests include spread spectrum communications, multiple access, and antijam techniques.

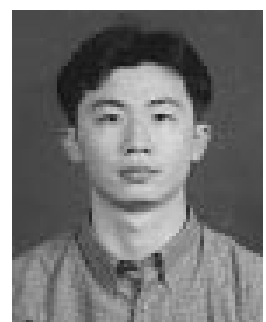

Chu-Ya Hsiao was born in Hsinchu, Taiwan in 1970. He received B.S. and M.S. degrees in communication engineering from the National Chiao Tung University, Hsinchu, Taiwan, in 1993 and 1995, respectively.

$\mathrm{He}$ is currently with Novatek Microelectronics, Hsinchu, Taiwan. His research interests include wireless access technologies, modulation, and coding theory. 